\title{
Terres cuites votives de Medma
}

Recherches sur le matériel des fouilles Orsi. Campagne 2020.

Documentation et analyse préliminaire de la technique de fabrication.

\section{Ágnes Bencze and Péter Véninger}

\section{(2) OpenEdition}

\section{Journals}

\section{Electronic version}

URL: http://journals.openedition.org/cefr/4907

DOI: $10.4000 /$ cefr.4907

ISSN: 2282-5703

\section{Publisher}

École française de Rome

\section{Electronic reference}

Ágnes Bencze and Péter Véninger, «Terres cuites votives de Medma ", Chronique des activités archéologiques de l'École française de Rome [Online], Italie du Sud, Online since 12 December 2020, connection on 14 December 2020. URL : http://journals.openedition.org/cefr/4907 ; DOI : https:// doi.org/10.4000/cefr.4907

This text was automatically generated on 14 December 2020.

(c) École française de Rome 


\section{Terres cuites votives de Medma}

Recherches sur le matériel des fouilles Orsi. Campagne 2020.

Documentation et analyse préliminaire de la technique de fabrication.

Ágnes Bencze and Péter Véninger

\section{AUTHOR'S NOTE}

Les recherches conduites depuis 2017 dans le cadre du programme « Terres cuites votives de Medma » sont soutenues par l'École Française de Rome, l'Université Catholique Péter Pázmány de Budapest et l'Accademia Nazionale dei Lincei, en partenariat avec le Musée Archéologique National de Reggio Calabria, la Surintendance ABAP de Reggio Calabria et Vibo Valentia et le Musée des Beaux-Arts de Budapest. La campagne de l'an 2020, de durée réduite à cause de la crise sanitaire, s'est déroulée entièrement dans les réserves du Musée Archéologique de Rosarno, sous la surveillance institutionnelle de Fabrizio Sudano, responsable de la Surintendance ABAP.

L'équipe dirigée par Ágnes Bencze (Université catholique Péter Pázmány) avait comme collaborateurs Péter Véninger (céramiste et restaurateur de céramiques antiques), Lili Tóth (ancienne élève de la Central European University, Budapest), András Sári et Bence Holnapy (étudiants, Université catholique Péter Pázmány). L'aspect informatique était pris en charge par Franco Prampolini, directeur du Laboratoire SuMMA, Università di Reggio Calabria et Antonio Gambino, informaticien-chercheur de la même institution.

\section{État de la recherche et objectifs de la campagne 2020}

1 En juillet et août 2020, notre équipe a poursuivi les travaux du projet « Terres cuites votives de Medma", portant sur la documentation et l'analyse systématique du matériel coroplastique votif trouvé dans la grande favissa (dépôt votif) du site Calderazzo (Rosarno) (fig. 1 et 2) ${ }^{1}$.

Compte tenu des conditions particulières imposées par la crise sanitaire, ainsi que de l'état de l'avancement de notre travail de documentation, nous avons établi pour la 
campagne de cette année des objectifs liés au perfectionnement de notre technique de documentation. Les trois axes principaux sur lesquels nous avons commencé à travailler étaient (1) la documentation photogrammétrique en 3D, (2) la transformation de notre recueil de données en une vraie base numérique appropriée à la gestion des données aussi en termes de recherche statistique et (3) l'établissement d'un système complexe et approfondi d'observations de caractère technique, susceptible d'apporter des informations pertinentes pour le classement du matériel. Il s'agit de trois démarches méthodologiques qui se complètent, voire se présupposent réciproquement et qui sont indispensables pour nous rapprocher, mieux qu'on pouvait le faire jusqu'ici, des anciennes questions concernant l'identité et l'organisation de l'atelier ou des ateliers responsables de la production des terres cuites votives de Calderazzo. Il faut rappeler en effet que l'une des questions centrales de la recherche consacrée à ce matériel est celle de la connexion des artisans et des dédicants de Medma avec ceux des autres cités de la "sphère locrienne ", Locres et Hipponion, durant les premières phases de fréquentation, c'est-à-dire de la deuxième moitié du VI ${ }^{\mathrm{e}}$ siècle aux décennies 450-430 av. J.-C. Les observations enregistrées jusqu'à nos jours, soit par notre équipe, soit par nos prédécesseurs, pour la plupart faites à l'œil nu, mais parfois avec une approche archéométrique préliminaire, ont déjà fait ressortir des particularités qui suggèrent des pratiques d'échanges et de collaboration systématique entre plusieurs centres créateurs durant cette période ${ }^{2}$. Pour cette campagne, notre objectif était de définir des critères d'observation plus détaillés concernant le procédé de fabrication, en prenant en considération toutes les étapes, dont on peut reconnaître les traces sur les objets, de la préparation de l'argile à la cuisson (à l'exception du finissage par peinture, puisque les terres cuites de Calderazzo ont conservé très peu de traces de couleurs).

Fig. 1 - Fragments de terres cuites dans les réserves du Musée Archéologique de Rosarno.

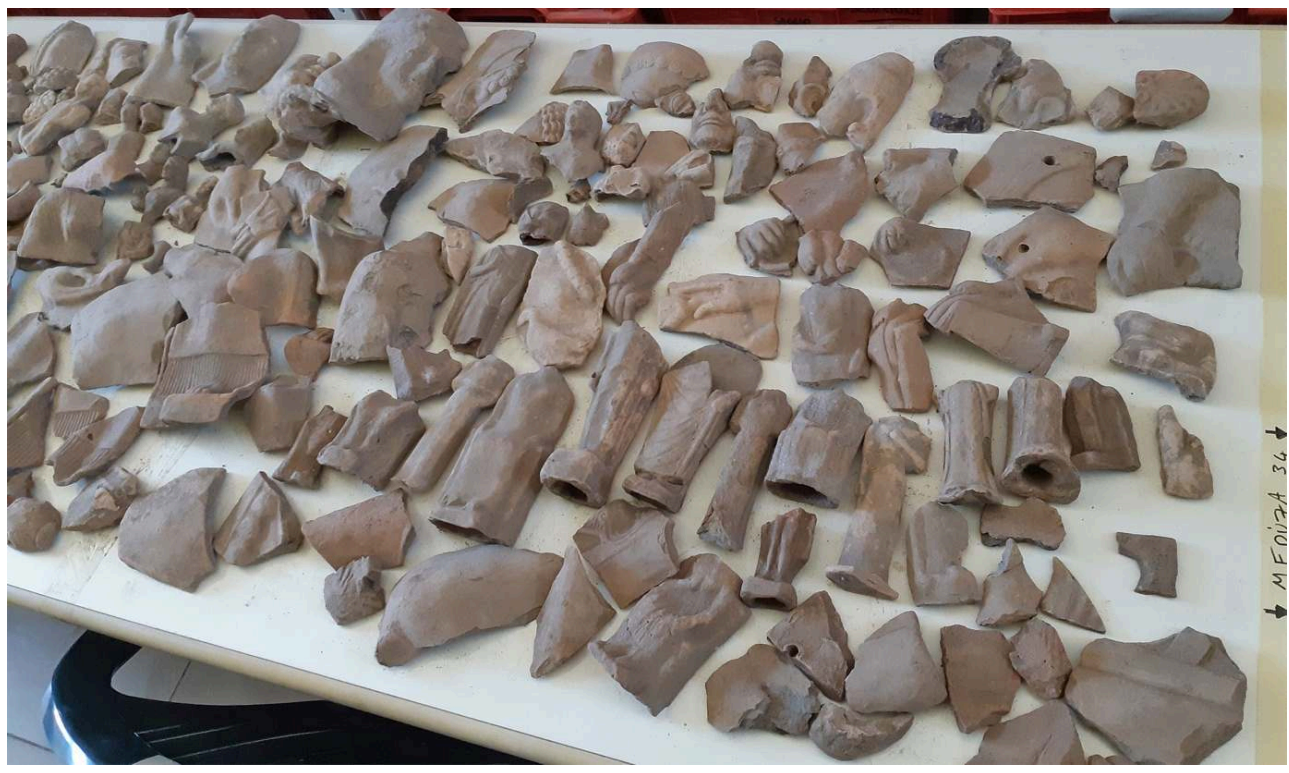

Crédit : Ágnes Bencze. 
Fig. 2 - Travail de classification de terres cuites dans les réserves du Musée Archéologique de Rosarno.

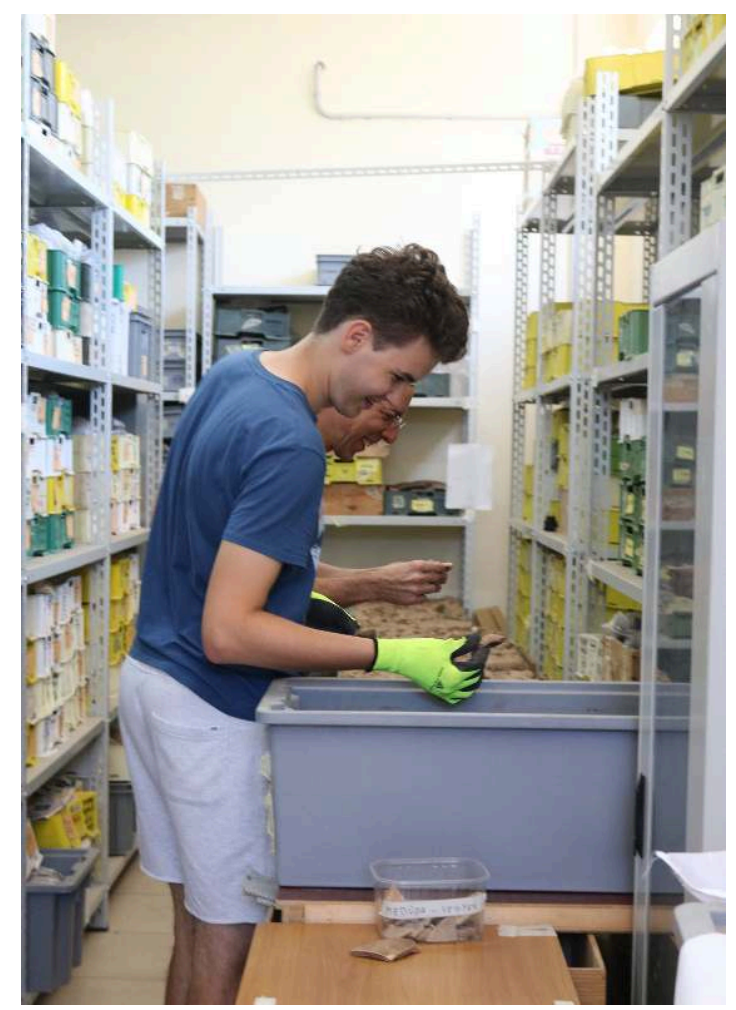

Crédit : Lili Tóth.

Une fois établies les catégories de description les plus importantes du point de vue technique, ceci nous permettra d'identifier les phénomènes véritablement distinctifs, susceptibles de définir des traditions d'atelier diverses à l'intérieur du matériel. En fait, ces observations sont préparatoires et complémentaires aux résultats de futures analyses pétrographiques qui, tout en étant significatifs en tant que données exactes, ne sont pas forcément décisifs en eux-mêmes pour la définition et la localisation des ateliers dont les produits sont parvenus au dépôt votif ${ }^{3}$. En même temps, les propositions qui seront présentées ci-dessous contribuent aussi à l'amélioration du système descriptif des fiches de notre base de données, ce qui permettra une appréciation plus exacte des proportions des diverses «fabriques " reconnues à l'intérieur de la documentation. Enfin, une définition plus précise de ces catégories descriptives nous fournit un outil qui permettra d'inclure « de plein droit » les pièces très fragmentaires dans l'évaluation statistique de notre matériel, en complétant les résultats qui peuvent être obtenus uniquement par le classement stylistique des objets les mieux conservés. Il faut remarquer en même temps - et nous tiendrons à l'indiquer ponctuellement au cours de l'exposé qui va suivre - que les raisonnements basés sur l'observation des traces de fabrication sont souvent insuffisants en eux-mêmes pour arriver à des conclusions définitives et seront à compléter par les résultats fournis par la géologie, la pétrographie et par le classement stylistique et typologique. Ce qui est proposé ici n'est par conséquent que la grille de description de départ, qui sera à affiner par la suite. 


\section{Caractéristiques techniques du matériel provenant du dépôt votif du site de calderazzo et propositions de critères de description}

\section{Remarques générales}

Une caractéristique importante du répertoire du dépôt votif de Calderazzo est la très grande variété des formes, compte tenu du fait que nous avons affaire à une production basée sur la reproduction en série, à l'aide de moules. La très grande variété formelle montre que les producteurs de ces terres cuites travaillaient avec un grand nombre de prototypes indépendants, dont ils exécutaient un nombre relativement restreint de moulages 4 .

5 Il est remarquable que les types de cette production n'étaient pas apparemment reproduits en plusieurs générations dérivées. À l'état actuel de la documentation on connaît une ou, plus rarement, deux générations de chaque type, mais les grandes séries dérivées sont absentes. Du point de vue des dimensions trois classes majeures sont à distinguer : les grands bustes, dont les plus gros exemplaires complets arrivent à $45 \mathrm{~cm}$ de haut, avec la tête proche de $20 \mathrm{~cm}$; les statuettes debout ou assises de 30 à $40 \mathrm{~cm}$ haut, la tête de 10 à $15 \mathrm{~cm}$; en dernier lieu les statuettes « miniatures » avec la tête de 3-4 cm. L'observation attentive des formes montre toutefois qu'il n'y a pas de rapport de dérivation mécanique entre les exemplaires de ces trois groupes, autrement dit, les statuettes les plus petites ne dérivent jamais des prototypes des statuettes moyennes, et les têtes utilisées pour celles-ci ne dérivent jamais de celles des bustes.

6 Un autre trait particulier de ce matériel est la très haute qualité du travail de modelage, à la fois en ce qui concerne la qualité esthétique des prototypes (autant qu'on puisse en juger d'après l'apparence de leurs reproductions moulées) et la réalisation soigneuse des pièces individuelles, au moins en ce qui concerne leur surface. En fait, en contraste avec les reliefs très précis et soignés des parties visibles, l'intérieur des pièces moulées dénote en général un travail plutôt négligeant, avec des «fautes» de fabrication qui devaient les rapprocher parfois au risque d'éclater au cours de la cuisson. Apparemment les coroplathes de Medma étaient très soucieux de la qualité esthétique de leurs produits, et beaucoup moins intéressés par la perfection technologique et par la routine « industrielle » de la fabrication.

7 Ces observations convergent à définir un cadre général qui caractérise les producteurs de ces terres-cuites comme une communauté d'artisans créateurs, qui comptait plusieurs personnalités de modeleurs individuels, sans pouvoir exclure la possibilité qu'ils aient été eux-mêmes les reproducteurs de leurs types. L'atelier responsable de la création des offrandes de Calderazzo se distinguerait ainsi parmi les centres producteurs de terres cuites du monde grec archaïque récent et classique ${ }^{5}$.

\section{Préparation de l'argile : dégraissants et impuretés non intentionnelles}

Nous tenons à préciser d'emblée que les observations qui suivent ne sont pas à confondre avec les résultats d'une analyse pétrographique. Une telle analyse, qui selon les standards actuels requiert une série d'examens complémentaires, exécutés à l'aide 
d'équipements scientifiques, sera indispensable à la conclusion du travail de documentation et de classification qui est en cours et devra faire partie du programme des campagnes à venir. L'observation à l'œil nu des caractéristiques de la pâte des terres cuites, déjà tentée par R. Miller en 1983 à titre d' " examens minéralogiques préliminaires » conduit, aujourd'hui comme avant, à distinguer en gros trois qualités d'argile, qui se distinguent par leurs couleurs et la variété des inclusions qu'elles renferment ${ }^{6}$. Le travail de documentation systématique d'un plus grand nombre de documents provenant du même dépôt votif fait toutefois émerger beaucoup de cas incertains, qui montrent la nécessité de l'analyse pétrographique de la composition chimique de nos artefacts pour vérifier l'hypothèse, en soi très tentante, de la coexistence de trois groupes de pâtes diverses dans le répertoire. Les résultats que l'on peut espérer de ces analyses resteront d'ailleurs insuffisants pour déterminer la provenance des argiles utilisées par les trois «fabriques». Pour cela il faudra les confronter avec une base de données géologique susceptible de localiser les diverses qualités d'argile sur la carte de la Calabre méridionale. Il faut de plus remarquer que la couleur des terres cuites dans leur état actuel est déterminée par une série de facteurs, parmi lesquels la composition chimique n'est pas le plus important. Au contraire, la couleur d'un objet de terre cuite dépend surtout des conditions de sa cuisson, un point sur lequel nous reviendrons par la suite.

Les observations suivantes n'enregistrent que des propriétés physiques, visibles à l'œil nu, utiles pour détecter les démarches adoptées par les artisans. En suivant l'ordre du procédé de la fabrication, nous traitons ici en premier lieu la préparation de l'argile, c'est-à-dire sa purification intentionnelle et l'ajout de composants qui pouvaient en modifier le comportement lors des autres phases de la fabrication des objets votifs.

Dépuration: La présence d'inclusions visibles de n'importe quelle composition (mica, oxyde de fer, chaux) exclut que l'argile ait été purifiée par la démarche de décantation connue aux potiers. Dans le cas des terres cuites trouvées dans le dépôt de Calderazzo c'est toujours le cas.

11 Dégraissants : Les dégraissants ${ }^{7}$ sont des grains de minéraux contenus dans l'argile, qui la rendent "moins grasse » et en modifient les qualités plastiques. Plus l'argile est "grasse ", plus elle est facile à modeler, tandis que l'argile dégraissée tend à devenir plus rigide est moins façonnable. En revanche elle a des meilleures propriétés techniques en ce qui concerne les étapes de fabrication successives: le séchage et la cuisson. Au cours du séchage, elle se rétrécit moins, mais plus rapidement et, par conséquent, une argile dégraissée pouvait sembler plus appropriée à la fabrication par moulage dans un contexte de production où cette technique était régulièrement employée, mais n'était pas encore développée au niveau d'une routine quasiindustrielle. Bien que la présence de dégraissants dans l'argile soit plus souvent naturelle que le résultat d'une adjonction intentionnelle, dans un cas comme celui des terres cuites de Calderazzo, il semble probable qu'elle correspondait à un choix conscient de part des artisans.

12 La pâte de nos terres cuites apparaît en effet fortement dégraissée et donc moins façonnable, plus appropriée à la fabrication d'objets plutôt grands et de structure plutôt simple, mais avec un très bon niveau de précision et de sécurité. La variété de dégraissants qui s'observent à l'œil nu montre une certaine diversité, dans les termes déjà enregistrés approximativement dans le passé, qui pourront permettre d'établir des groupements scientifiquement définis à la suite d'analyses pétrographiques. Dans le cas 
de la plus grande partie des objets, la pâte semble être homogène, avec une forte présence de mica (probablement naturelle) et de grains de quartzite. En revanche, les terres cuites du dépôt ne semblent pas en général contenir de la chaux (fig. 3).

Fig. 3 - Détail de statuette de terre cuite, pâte riche de grains blancs, probablement du quartzite, en fonction de dégraissant. Musée Archéologique de Rosarno.

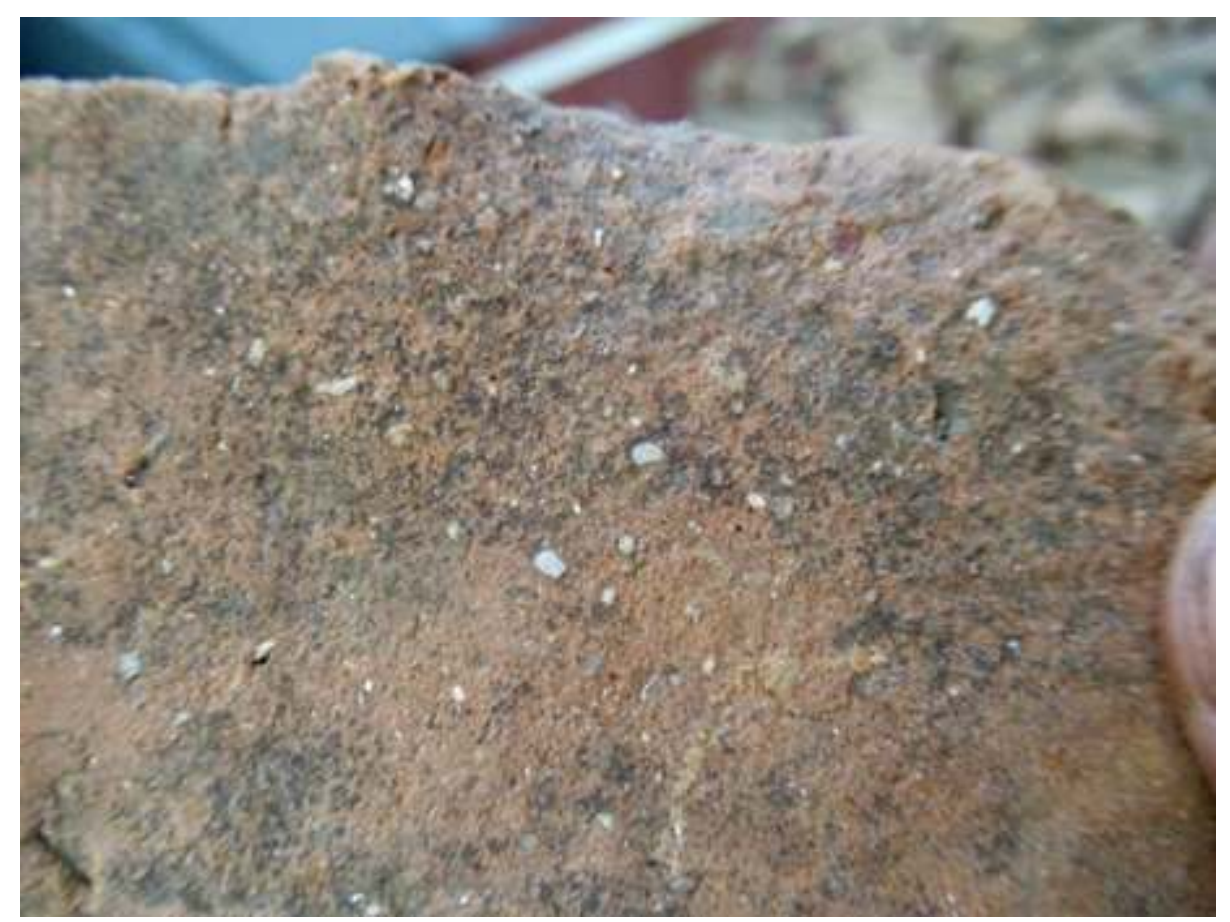

Crédit : Péter Véninger.

13 Un autre groupe de terres cuites est caractérisé par la présence moins homogène de dégraissants, tout en contenant du mica, tandis qu'elles contiennent des grosses inclusions de couleurs, formes et dimensions variables, qui sont à considérer probablement comme des impuretés non intentionnelles, restées dans la pâte faute d'un traitement de purification efficace.

15 Inclusions non intentionnelles: en effet, il convient de distinguer cette catégorie d'inclusions des dégraissants, surtout dans le cas où la quantité occasionnelle ou la distribution très irrégulière des minéraux exclut qu'ils aient eu une quelconque fonction prévue par les artisans. Dans le répertoire de Calderazzo on peut observer au moins les cas suivants :

- Traces de restes organiques brûlés : généralement il s'agit d'un trou de forme anguleuse sur la surface (fig. 4). 
Fig. 4 - Trace de restes organiques sur la surface d'une terre cuite. Musée Archéologique de Rosarno.

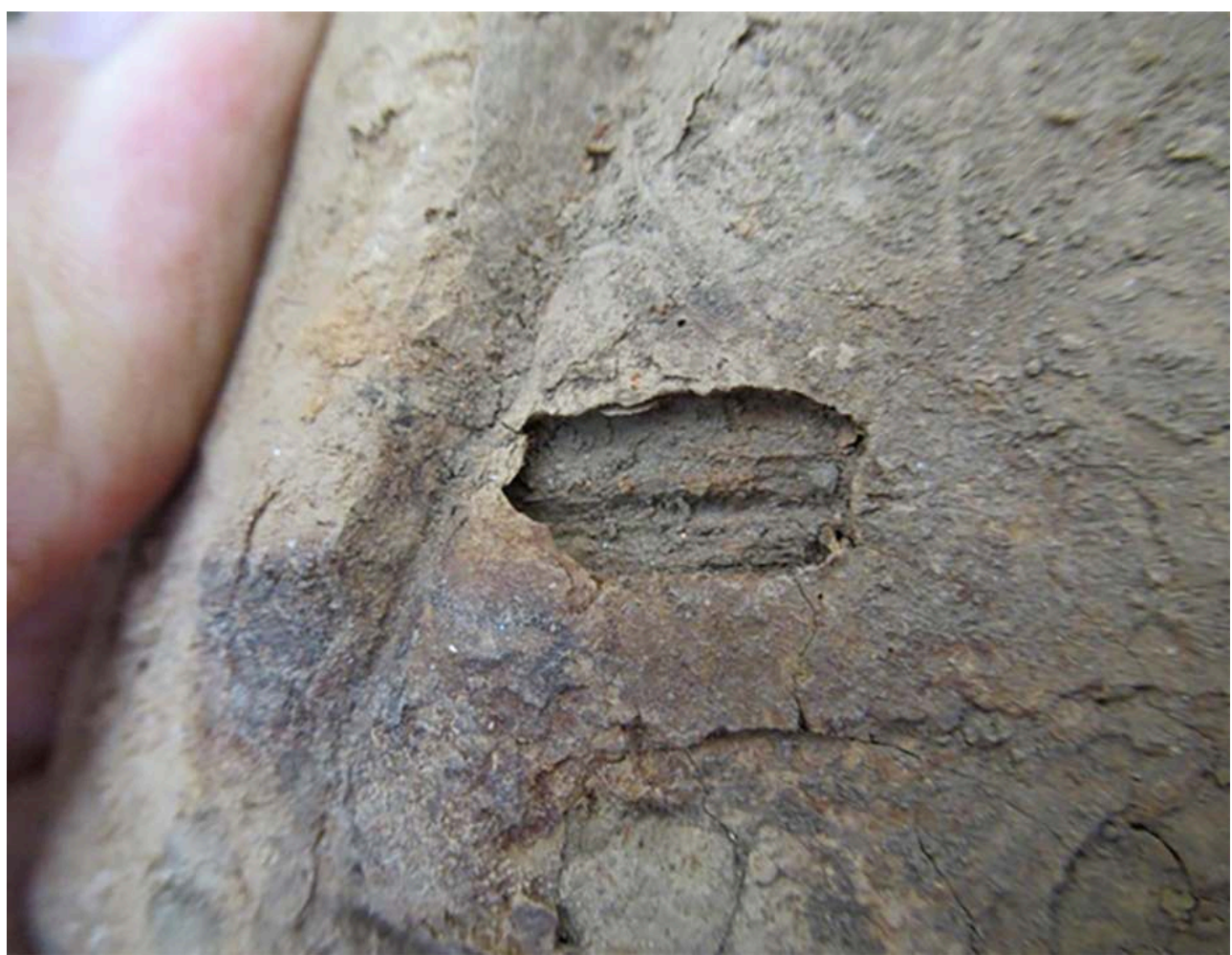

Crédit : Péter Véninger

- Caillou : on appellera ainsi toute inclusion dépassant $3 \mathrm{~mm}$. La présence de cailloux dans l'argile comporte un risque constant de fêlure, seule les pâtes fortement dégraissées sont susceptibles de les soutenir sans se fendre. Dans le répertoire de Calderazzo, nous en trouvons des exemples extrêmes dans les terres cuites moins homogènes, ce qui confirme aussi la constatation précédente, c'est-à-dire que nous avons affaire pour la plupart à des pâtes fortement dégraissées (fig. 5). 
Fig. 5 - Grand caillou inclus dans le nez d'un buste de terre cuite. Musée Archéologique de Rosarno.

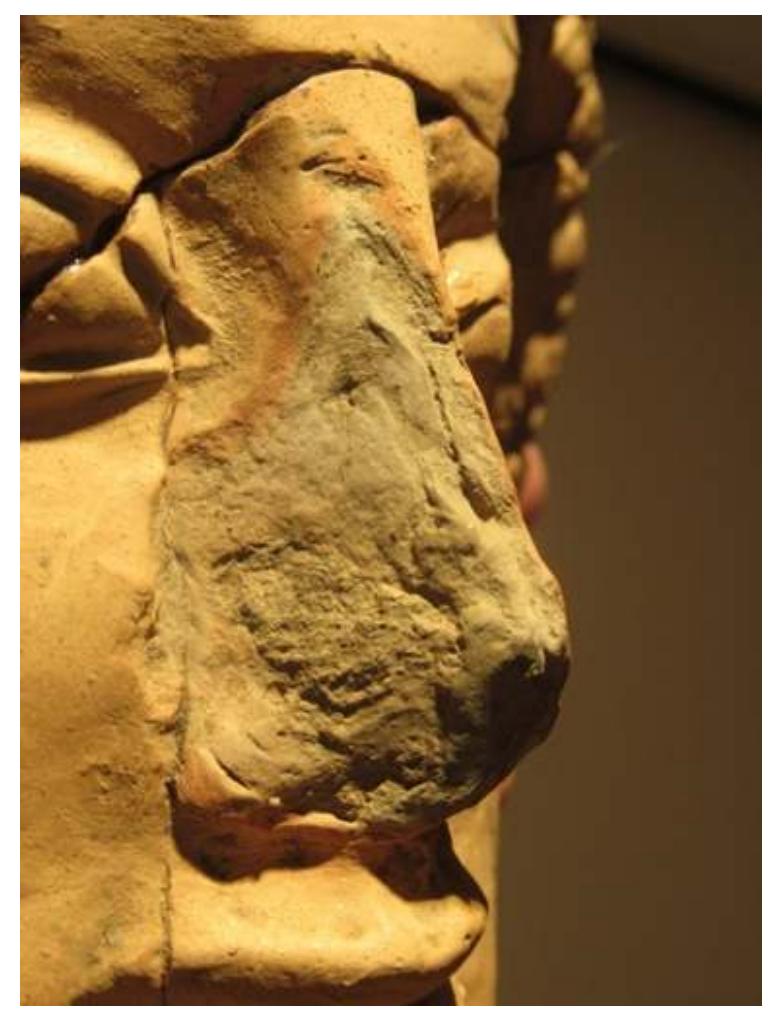

Crédit : Péter Véninger.

- Oxyde de fer en forme de grandes inclusions (fig. 6). 
Fig. 6 - Inclusion d'oxyde de fer sur la surface d'une protomé de terre cuite. Musée Archéologique de Rosarno.

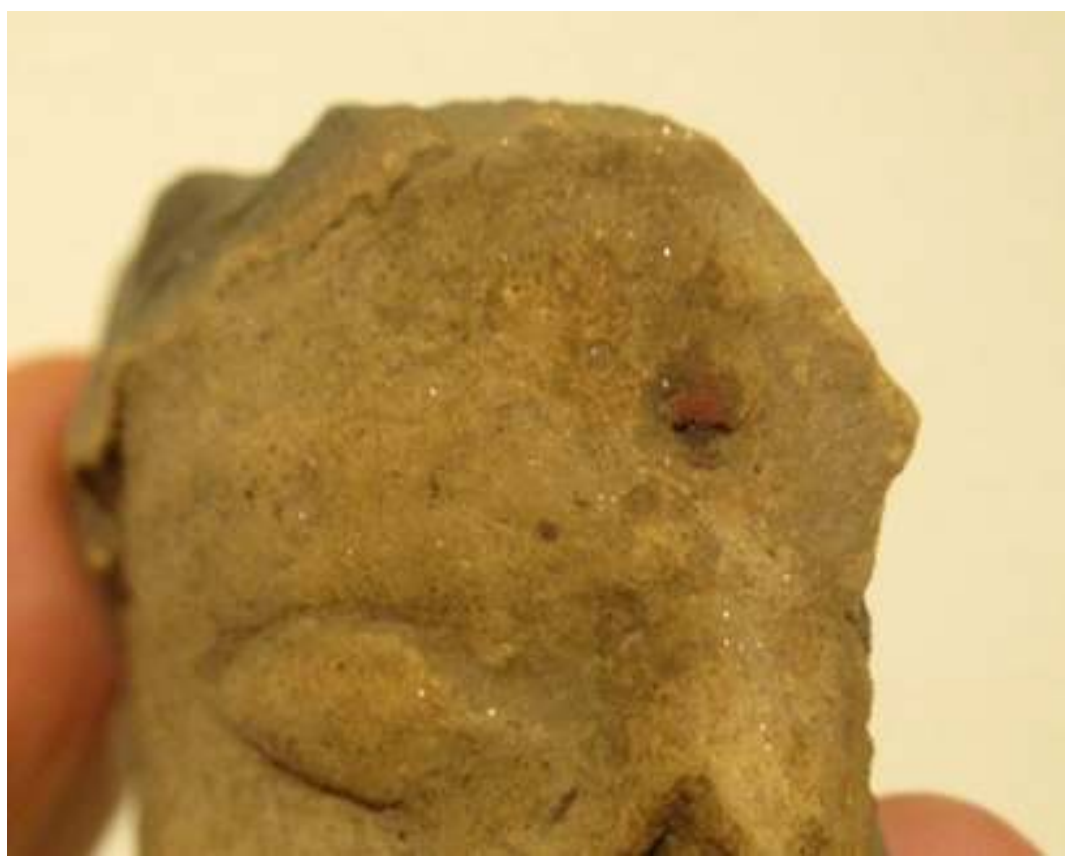

Crédit : Péter Véninger

- Un autre type de minéral, qui se trouve souvent dans les terres cuites à pâte stratifiée (voir ci-dessous) et qui n'est pas à confondre avec l'oxyde de fer, en dépit de sa couleur rouille. Ces inclusions tendent à être plus larges $(1-3 \mathrm{~mm})$ et causent des brisures en forme de cratère qui s'étendent depuis les couches profondes jusqu'à la surface de la terre cuite ${ }^{8}$ (fig. 7 et 8).

Fig. 7 - Inclusion « en cratère » sur la surface d'une statuette de terre cuite. Musée Archéologique de Rosarno.

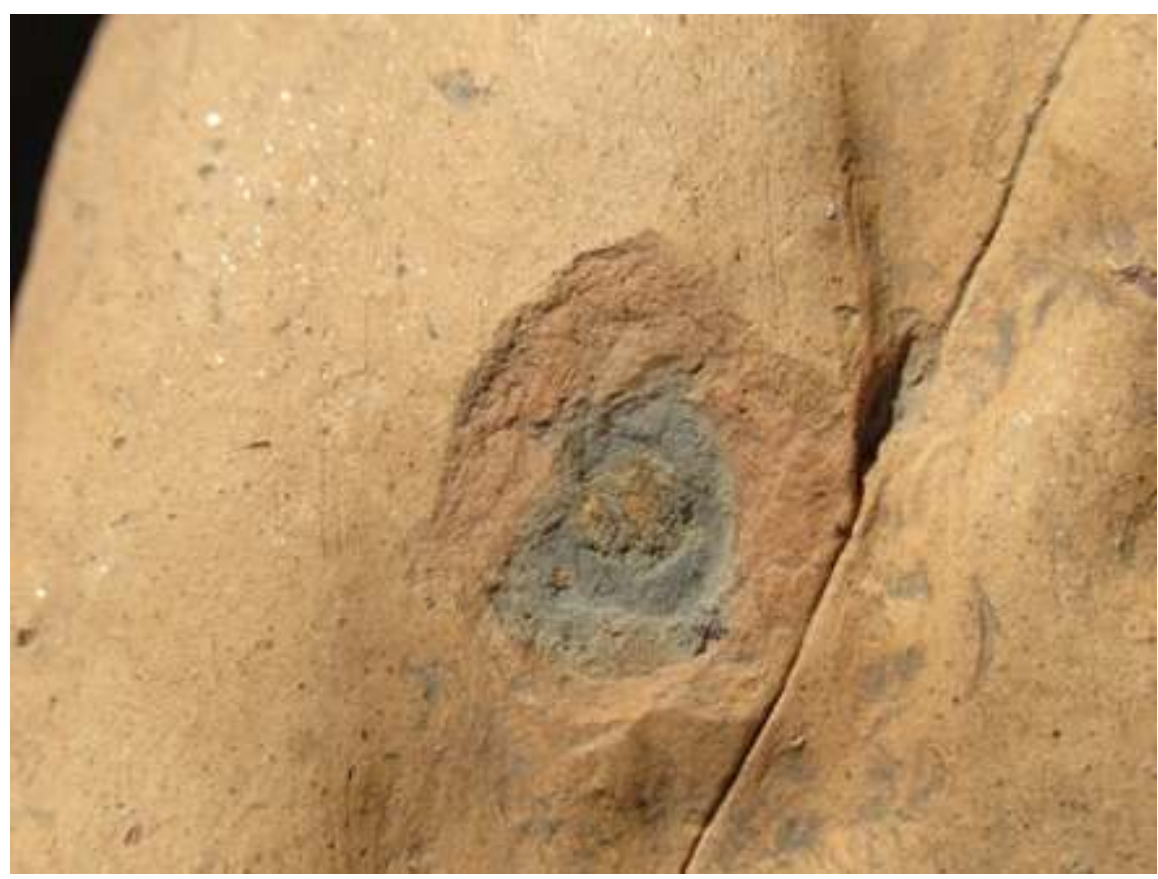

Crédit : Péter Véninger. 
Fig. 8 - Inclusion « en cratère » sur la surface d'une statuette de terre cuite. Musée Archéologique de Rosarno.

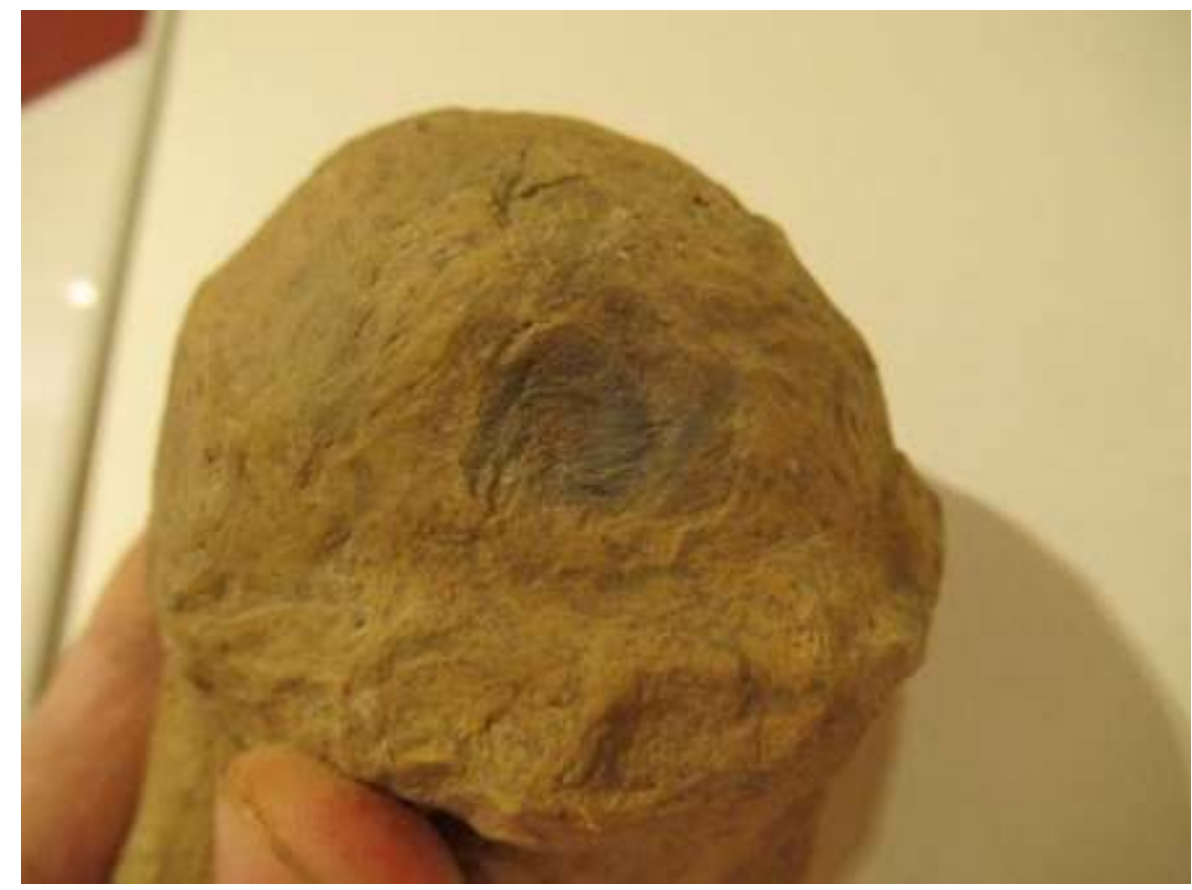

Crédit : Péter Véninger.

La détermination exacte de la composition chimique de ces inclusions, en particulier du dernier type, dont l'apparition est très régulièrement liée à une qualité de pâte spécifique, serait très utile pour la distinction et, éventuellement, pour la localisation des argiles utilisées dans le matériel de Calderazzo.

\section{Consistance de l'argile au moment du moulage}

Contrairement à ce qu'on vient de dire de la composition chimique de l'argile, une autre propriété, le taux d'humidité au moment du moulage, ne peut être montrée par les analyses pétrographiques. En revanche, elle se reconnaît très bien en observant les traces de fabrication et elle est un indice très pertinent, puisqu'elle est liée directement à la méthode de travail des artisans. Par conséquent ces observations, prises en considération l'ensemble avec les traces de modelage et moulage, sont susceptibles de nous rapprocher de la caractérisation d'un atelier.

Dans le répertoire de Medma nous trouvons deux groupes majeurs du point de vue de la consistance de l'argile utilisée pour le moulage :

- Un nombre plus restreint de terres cuites présente des traces de pressage survenu à un état extrêmement mouillé de l'argile, qui laisse des traces caractéristiques sur la face interne de la croûte (fig. 9 et 10).

- La plus grande partie des terres cuite de Calderazzo révèle l'usage d'une argile toujours tendre, mais plus proche de la consistance considérée comme «normale » dans la pratique actuelle (fig. 11). 
Fig. 9 - Traces de pressage sur la surface interne d'une terre cuite moulée d'argile extrêmement tendre (consistance « boueuse »). Musée Archéologique de Rosarno.

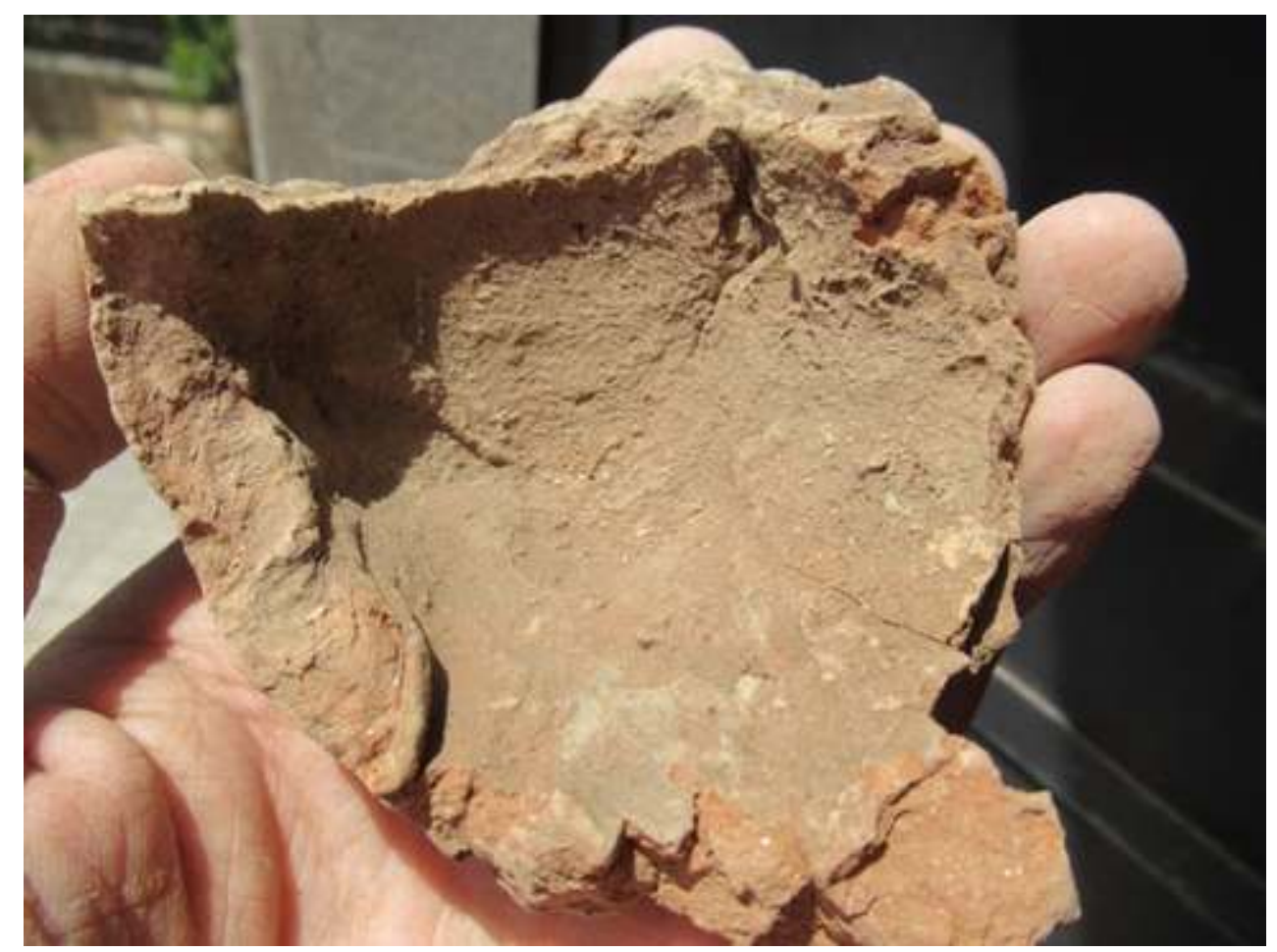

Crédit : Péter Véninger

Fig. 10 - Traces de pressage sur la surface interne d'une terre cuite moulée d'argile extrêmement tendre. Musée Archéologique de Rosarno.

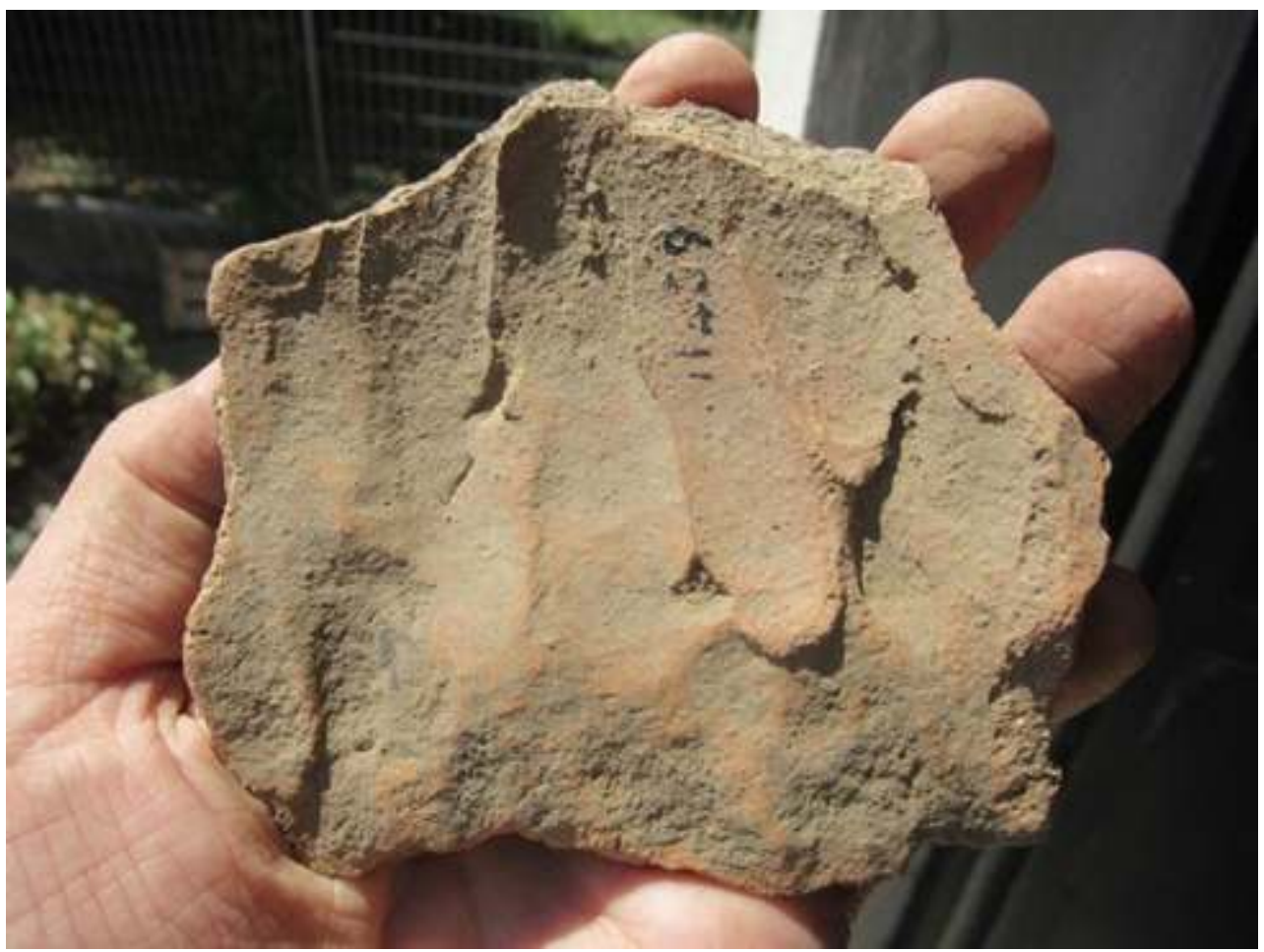

Crédit : Péter Véninger 
Fig. 11 - Traces de pressage sur la surface interne d'une terre cuite moulée d'argile tendre ou moyenne (presque « normale »). Musée Archéologique de Rosarno.

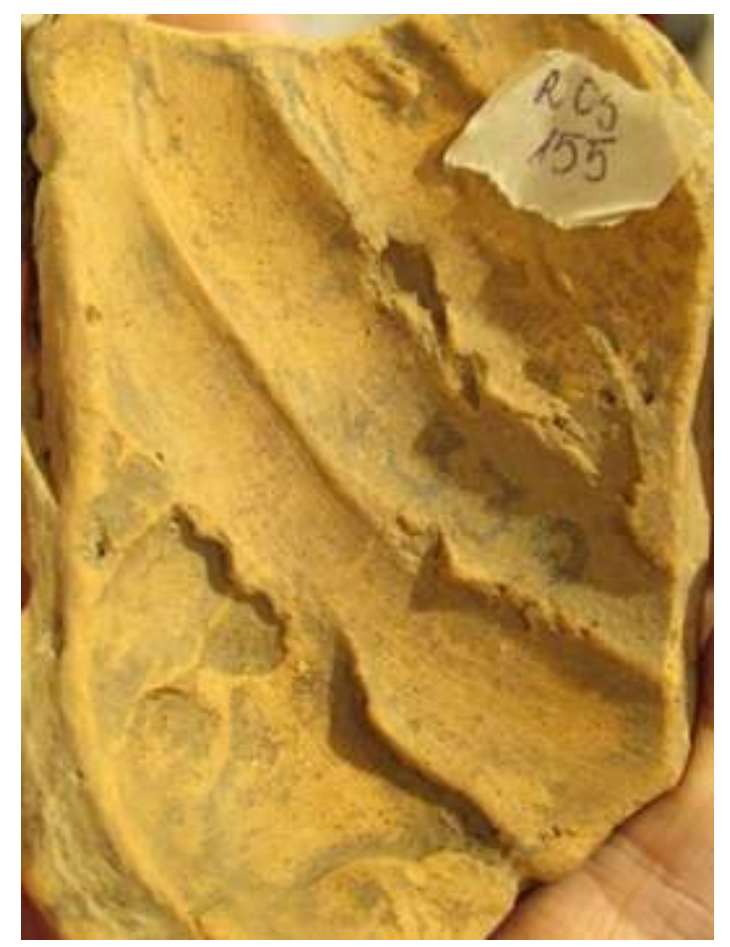

Crédit : Péter Véninger.

L'usage d'argiles très tendres, c'est-à-dire à très haut contenu d'eau, est plutôt fréquent dans les productions céramiques antiques, en comparaison avec la pratique des céramistes d'aujourd'hui. Ce phénomène est toujours lié au haut contenu de dégraissants, puisque l'humidité aidait les artisans à contrebalancer la rigidité de l'argile "maigre » durant le modelage ou le pressage. En même temps, l'argile fortement dégraissée permet à l'eau de s'évaporer plus rapidement et empêche que la forme modelée ne se déforme trop au cours du séchage. L'usage d'argiles très tendres est d'ailleurs typique du travail des potiers qui continuent à utiliser des techniques traditionnelles aujourd'hui encore. Nous avons eu occasion de documenter l'usage actuel d'une argile de consistance correspondant à notre deuxième groupe (tendre, bien que proche de la «normael») dans le voisinage de Rosarno, au quartier historique de potiers de Seminara (fig. 12). 
Fig. 12 - Tournage en argile tendre en 2020 à Seminara, Calabre.

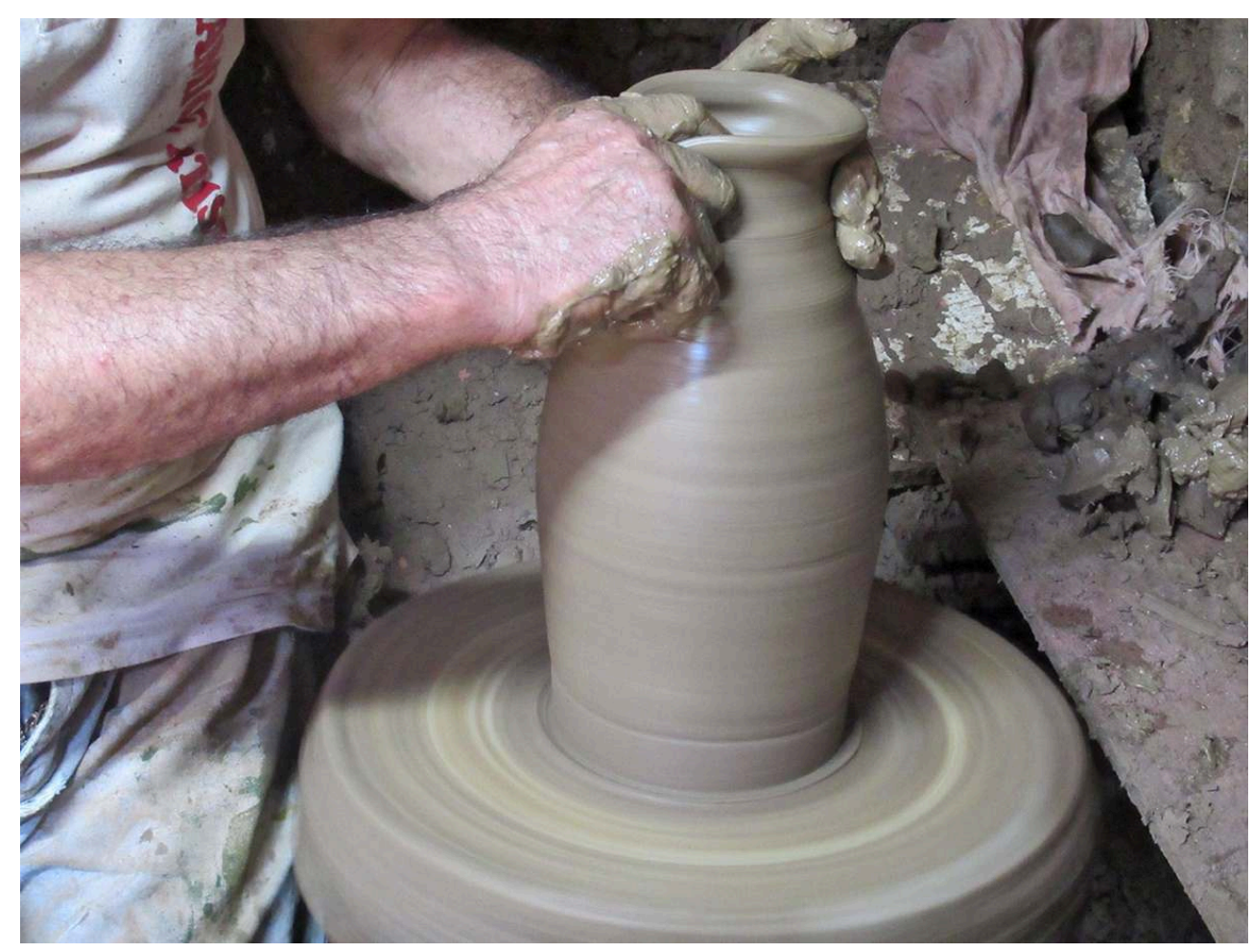

Crédit : Péter Véninter.

Tout compte fait, dans notre cas l'usage constant de l'argile à un état tendre ou extrêmement tendre, détecté dans la pratique des mouleurs des terres cuites de Calderazzo, confirme l'hypothèse formulée, à savoir le recours à des pâtes fortement dégraissées. Un exemple qui illustre bien l'apparence d'une terre cuite moulée avec une argile extrêmement tendre et probablement très riche en dégraissants est fourni par une protomé datant du VI ${ }^{\mathrm{e}}$ siècle av. J.-C. exposée au Musée de Rosarno (fig. 13). La comparaison systématique des observations concernant la consistance de l'argile avec la typologie stylistique de notre répertoire montrera probablement que l'usage de l'argile particulièrement humide au moulage fut abandonné graduellement au cours des décennies qui marquent la transition de l'époque archaïque à la première phase classique. 
Fig. 13 - Exemple de terre cuite moulée d'argile extrêmement tendre. Musée Archéologique de Rosarno.

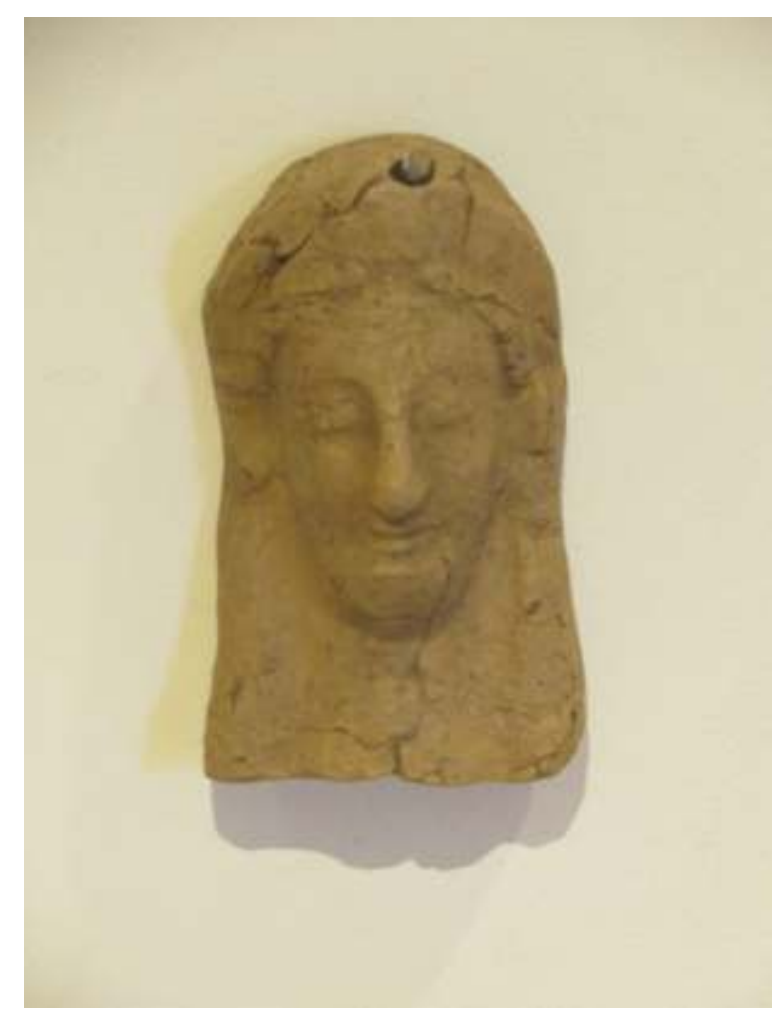

Crédit : Péter Véninger.

\section{Prototypes et moules}

Nous ne connaissons aucun prototype ni aucun moule conservé de cette production. Pour nous faire une idée de la qualité de la première création plastique et des outils de travail fabriqués directement à partir de celle-ci, nous devons nous appuyer sur le témoignage conservé par les pièces finies, pour la plupart fragmentaires, qui nous sont parvenues. Il faut préciser à ce propos qu'une grande partie de celles-ci ne permettent pas de jugement objectif à cause de l'état de conservation, sujet - à Medma, comme ailleurs - à toute une variété d'incidents corrosifs. En dépit de tous ces facteurs de perte d'information, nous pouvons exposer quelques observations et suppositions.

À travers le témoignage indirect des pièces conservées, on présume que les prototypes de la plupart des offrandes ensevelies à Calderazzo étaient de très haut niveau, en ce qui concerne la qualité plastique et la précision du modelage. Le même jugement peut être formulé aussi pour les moules (et pour la plupart de nos pièces il s'agit de la "première génération ", à en juger par les dimensions).

Les prototypes étaient, en outre, modelés d'une façon très " réfléchie ", tenant toujours compte des limites et des nécessités techniques imposées par le moulage. Les modeleurs faisaient attention, en fait, à la simplicité des formes, permettant de réaliser le moulage avec un seul moule, au moins en ce qui concerne la face frontale du volume principal de la composition. Les statuettes complètes de la première moitié $\mathrm{du}^{\mathrm{e}}$ siècle étaient reproduites généralement à l'aide d'un moule de front et d'une paroi d'arrière lissée sur une planche, très probablement de bois, comme le montrent les traces de 
nervures qu'on discerne sur quelques exemplaires (fig. 14). Les composantes étaient assemblées après le moulage avec de fréquents ajouts modelés, sur lesquels nous allons revenir. Les statuettes étaient, bien entendu, vides, avec la base ouverte en bas et souvent un grand trou d'assemblage découpé sur le dos ou aussi sur les deux côtés. Il fallait porter une attention particulière au cou des figures, qui étaient la partie la plus fragile de la plupart des compositions et posait également des problèmes spéciaux au moment du moulage, puisqu'il fallait absolument éviter d'enfermer de l'air dans la statuette passée à la cuisson - par exemple dans la tête vide, mais fermée par un cou éventuellement solide. Les artisans étaient évidemment conscients de ces problèmes et les affrontaient avec des solutions appliquées rigoureusement.

Fig. 14 - Dos de statuette, avec traces des nervures d'une planche de bois. Musée Archéologique de Rosarno.

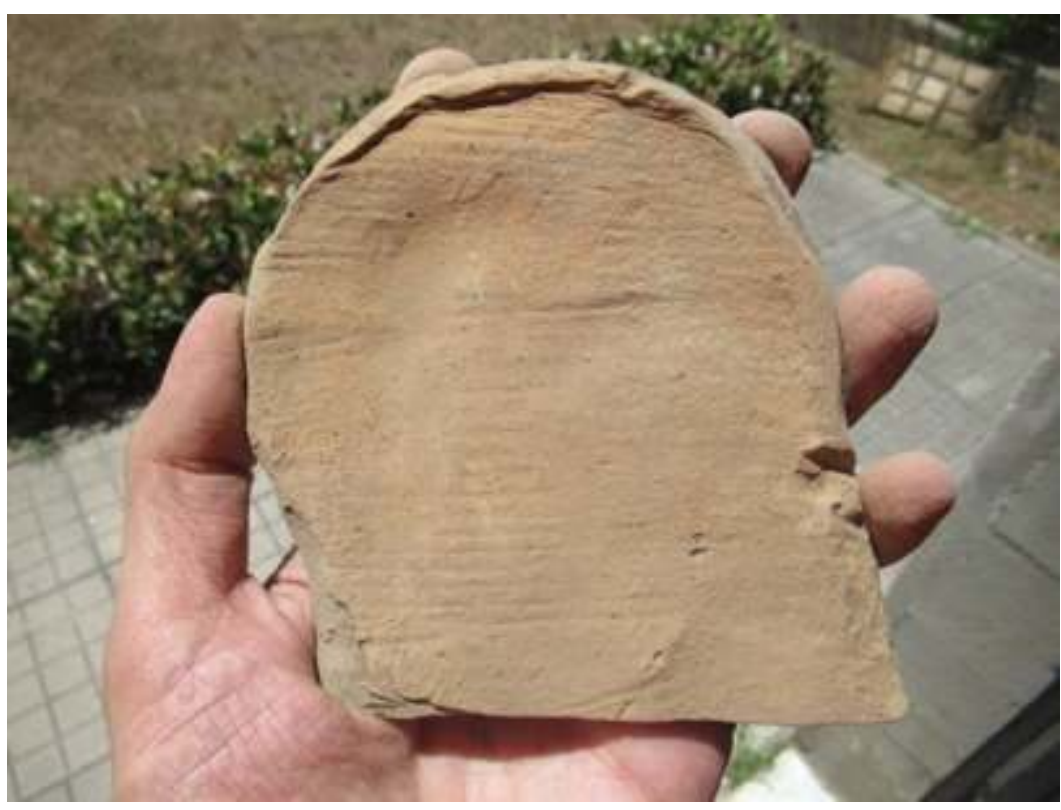

Crédit : Péter Véninger

Quant aux moules, à en juger toujours par la base des surfaces moulées qui en sont sorties, ils étaient également très bien faits, réalisés avec une attention particulière à la précision des détails. Les pièces moulées peuvent, en fait, révéler les défauts des moules eux-mêmes, mais, pour notre répertoire, ces malformations sont exceptionnelles et, dans les rares cas observés, minimales.

\section{Caractéristiques du moulage}

$\mathrm{Du}$ point de vue du travail de pressage, le trait spécifique le plus important du répertoire de Calderazzo est le contraste qu'on observe entre la qualité des surfaces externes, reproduisant très soigneusement les détails plastiques du prototype, et le caractère approximatif des surfaces internes, qui pullulent d'imperfections. Les artisans étaient évidemment intéressés par la qualité esthétique, mais beaucoup moins par la perfection technique, au point de se permettre aussi des fautes susceptibles de mettre en péril la stabilité de leurs produits. Ainsi les surfaces internes des pièces sont souvent «confuses » et l'épaisseur des parois très irrégulière. En revanche, ils étaient très soucieux de remplir les moules le plus parfaitement possible, pour ne perdre aucun 
détail plastique. C'est pour cette raison que la croûte est généralement très épaisse auprès du rebord et tend à s'amincir dans les parties les plus saillantes (et par conséquent les plus profondément creusées dans les moules).

Les terres cuites étaient moulées pour la plupart en pressant plusieurs couches de feuilles, formant la croûte, généralement deux ou trois. Les artisans tendaient à être peu constants aussi à cet égard : le nombre de couches n'est jamais identique partout sur une pièce moulée ; au contraire, il est évident que l'ajout d'une autre feuille servait généralement à renforcer la paroi où elle s'amincissait excessivement ou même à combler des lacunes, quand la feuille originale n'arrivait pas à couvrir le moule tout entier. Très souvent, les couches superposées sans précaution renferment des bulles d'air, et c'était un pur hasard si elles n'éclataient pas au cours de la cuisson.

Ces particularités caractérisent pratiquement l'ensemble du matériel, de façon constante et indépendamment des différences dans la qualité des pâtes. Puisque les observations techniques dénotent clairement une certaine attitude vis-à-vis du travail artisanal, en nous permettant de décrire une sorte de mentalité, l'homogénéité de ces phénomènes suggère aussi que les producteurs de ces offrandes, tout en utilisant diverses qualités d'argile et, probablement, diverses techniques de cuisson, partageaient les mêmes bases culturelles, sans doute parce qu'ils étaient formés dans la même tradition artisanale.

En examinant le revers des pièces moulées, on peut distinguer deux modalités principales quant à la technique de pressage de l'argile dans le moule: d'une part les chaines d'empreintes digitales contigües (fig. 15), de l'autre les traces du lissage en sillons parallèles (fig. 16).

Fig. 15 - Surface interne de pièce moulée. Musée Archéologique de Rosarno.

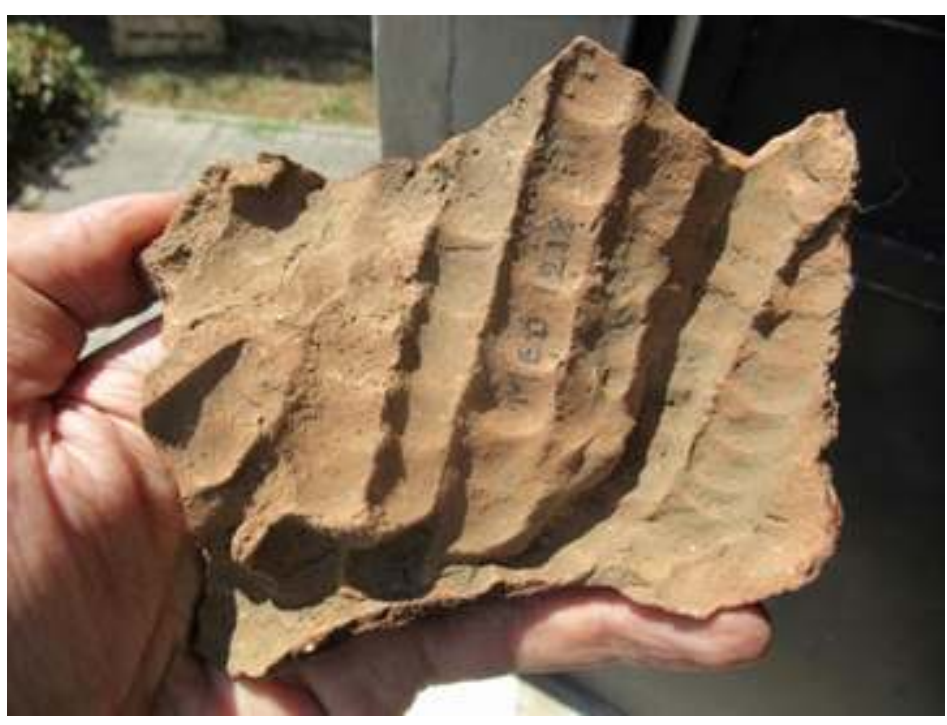

Crédit : Péter Véninger. 
Fig. 16 - Surface interne de pièce moulée. Musée Archéologique de Rosarno.

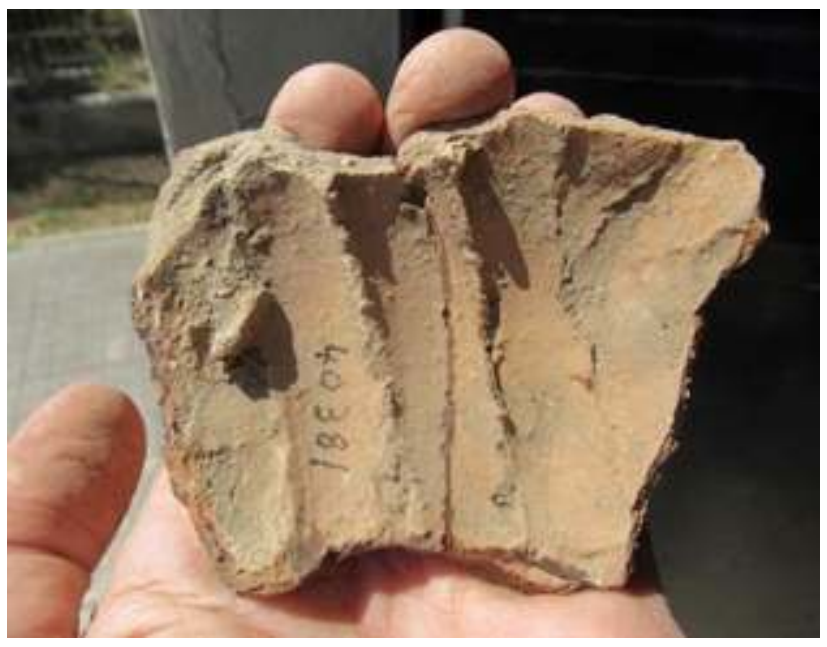

Crédit : Péter Véninger.

Parmi les fautes de moulage typiques nous pouvons mentionner :

- les « doubles contours » (se produisent lorsque le mouleur presse deux fois la croûte dans la moule, par accident) : une faute presque inexistante dans ce répertoire, un fait qui atteste l'attention particulière que les artisans prêtaient à la surface externe moulée ;

- le moule rempli imparfaitement: plutôt fréquent, mais ne se produit jamais dans une mesure importante ;

- le pressage excessif : très fréquent, ce qui dénote également le souci particulier pour la reproduction de la forme plastique ;

- la paroi trop fine ou trop épaisse : faute constante du répertoire qui conduit parfois à l'écrasement de l'objet moulé, lorsqu'un trait de parois est trop lourd pour se tenir en position avant la cuisson (fig. 17). 
Fig. 17 - Exemple de statuette écrasée par le poids de la croute trop épaisse avant la cuisson, vue de bas. Musée Archéologique de Rosarno.

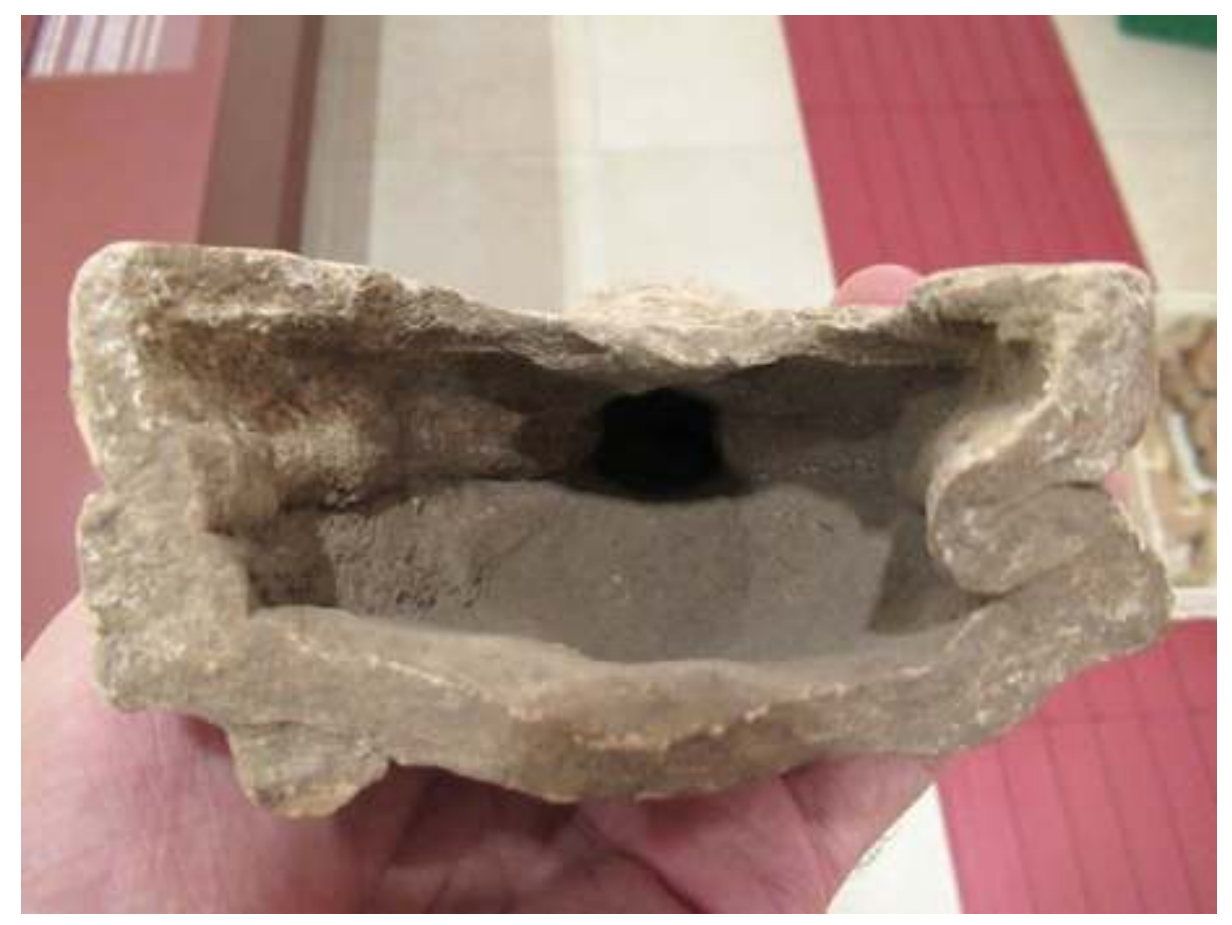

Crédit : Péter Véninger

\section{Assemblage, modelage et finissage des pièces moulées}

$\mathrm{Au}$ sein de la production attestée dans le dépôt votif de Calderazzo, les opérations secondaires de façonnage et d'assemblage qui suivaient le moulage avaient une importance particulière, à tel point qu'on peut considérer cette pratique " hybride » comme un des traits les plus caractéristiques de leur technique de fabrication. Il s'agit, encore une fois, de démarches susceptibles de caractériser d'une manière déterminante toute une tradition d'atelier; ces phénomènes et leurs conséquences sur l'originalité créative et sur le rapport des "prototypes » aux « séries de moulage » ont été analysés en détail récemment ${ }^{9}$. Nous nous concentrerons ici seulement sur l'énumération des indices observés qui permettent de reconnaître une série de solutions techniques pratiquées par les artisans producteurs.

Assemblage de pièces fabriquées séparément : La pratique d'ajouter des éléments façonnés à la main, comme des boucles d'oreilles, des ornements de tête, plus rarement des barbes, des bras ou des attributs est attestée dès la phase la plus ancienne, au VIe siècle. La technique même d'insérer les ajouts montre, toutefois, une certaine variété et suggère qu'il est possible de tracer des processus d'évolution à cet égard.

Les statuettes étaient pour la plupart certainement moulées en deux parties majeures, tête et corps, et assemblées avant la cuisson, à l'état de " consistance cuir » (Müller) des pièces moulées. Cette pratique permettait de varier l'accouplement entre têtes et corps dès le début $d u \mathrm{~V}^{\mathrm{e}}$ siècle $^{10}$. Sur quelques pièces fragmentaires, on a pu observer les traces typiques de la préparation des surfaces d'argile encore crue pour l'assemblage : la fig. 18 montre par exemple un détail de surface pourvue d'un sillon creusé pour renforcer la solidité d'un élément ajouté (dans ce cas de la paroi de dos). 
Fig. 18 - Sillon creusé sur un trait de surface pour faciliter l'assemblage à l'état demi-sec. Musée Archéologique de Rosarno.

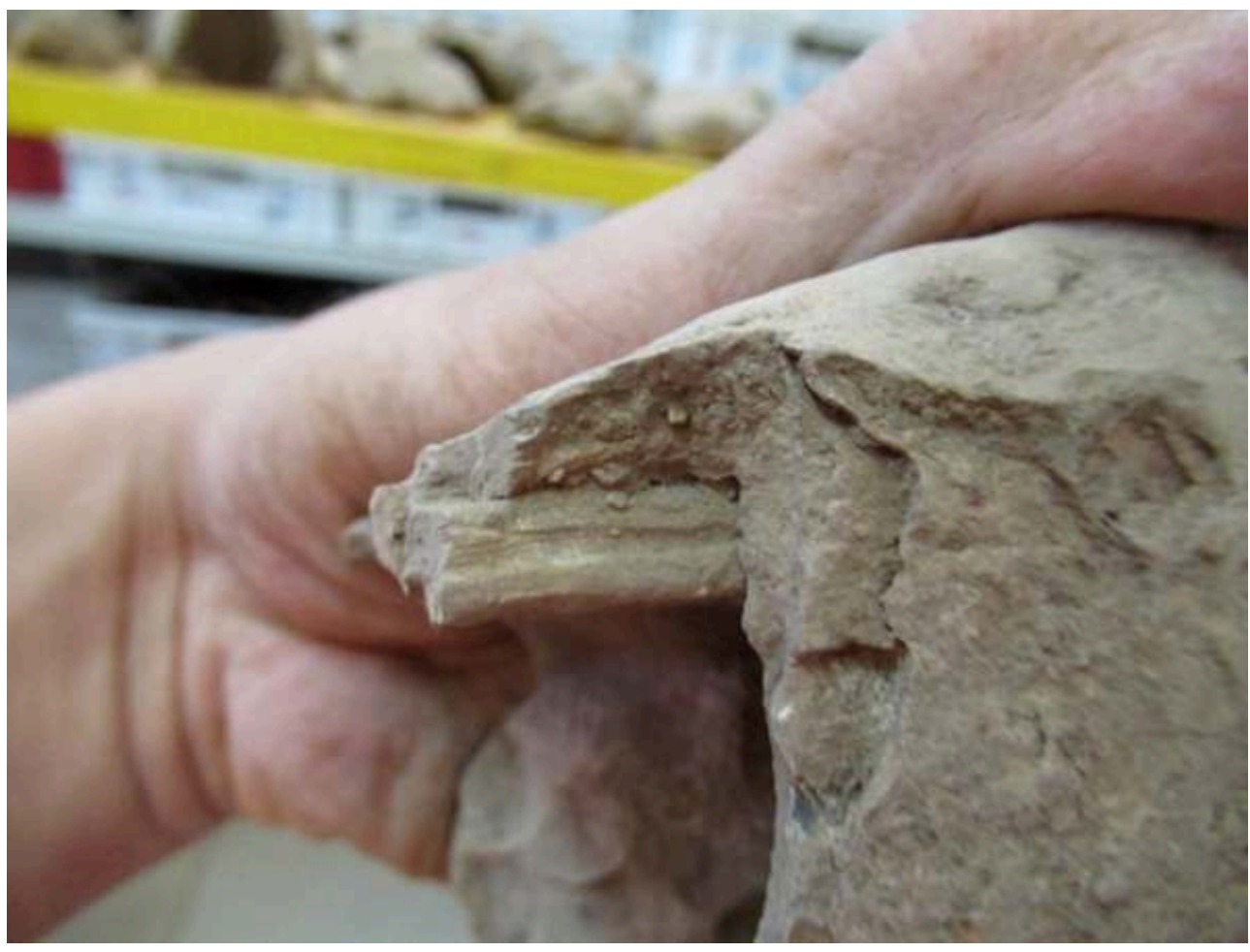

Crédit : Péter Véninger.

Plus rarement, l'ajout d'éléments façonnés séparément pouvait se produire même après la cuisson : c'était le cas des bras, associés en général à quelques types bien déterminés, dont on trouve aussi des exemplaires avec des surfaces ovales, lissées, soigneusement circonscrites, au niveau des coudes, où devaient être attachés les bras, cuits séparément, à l'aide de collages (fig. 19). 
Fig. 19 - Statuette avec des surfaces ovales lissées prévues pour l'ajout de bras après la cuisson. Musée Archéologique de Rosarno.

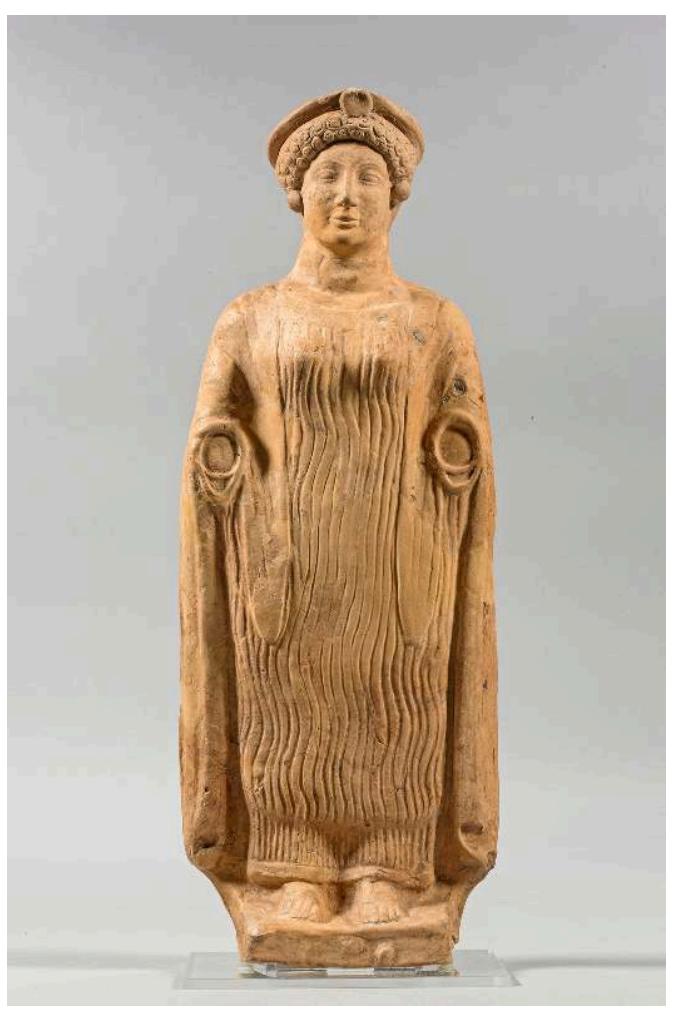

Crédit : László Mátyus.

Finissage par retouches et corrections: Les détails les plus finement élaborés étaient très souvent réalisés ou perfectionnés par une variété de procédés, comprenant le modelage à la main, à l'aide d'outils, l'estampillage et la construction par assemblage de plusieurs éléments moulés. La variété des formes et des techniques est la plus évidente dans le cas des chevelures. À part les " coiffures bouclées », souvent obtenues par une série de boucles estampillées, les chevelures « lisses ", à mèches ondulées parallèles, peuvent être réalisées, elles aussi, par l'ajout d'une masse de cheveux moulée séparément (fig. 20), ou par des retouches libres, exécutées sur la tête moulée, à l'aide d'un outil pointu laissant des traces moins régulières (fig. 21). Une tête fragmentaire, cassée de façon particulièrement heureuse du point de vue de notre enquête, permet de reconnaître les composants d'une construction soigneuse à partir d'éléments moulés et façonnés (fig. 22). 
Fig. 20 - Fragment de tête de statuette avec chevelure moulée à l'aide d'un moule partiel. Musée Archéologique de Rosarno.

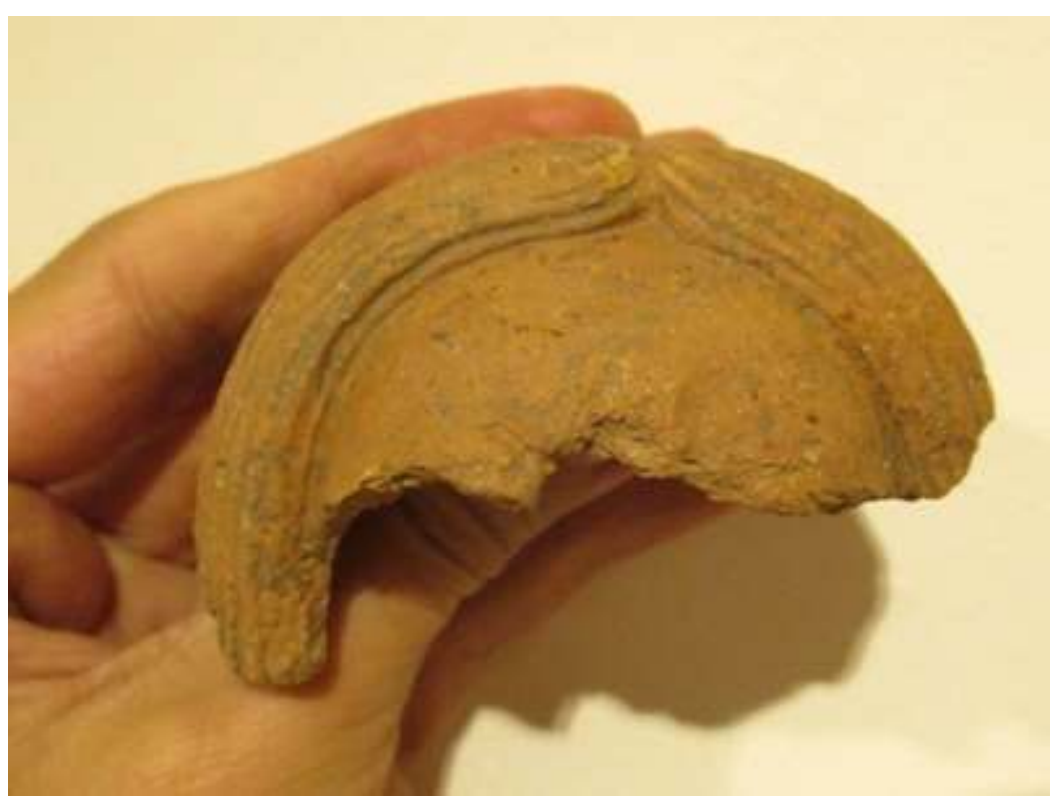

Crédit : Péter Véninger.

Fig. 21 - Fragment de tête de statuette avec chevelure pourvue de détails incisés à la main libre. Musée Archéologique de Rosarno.

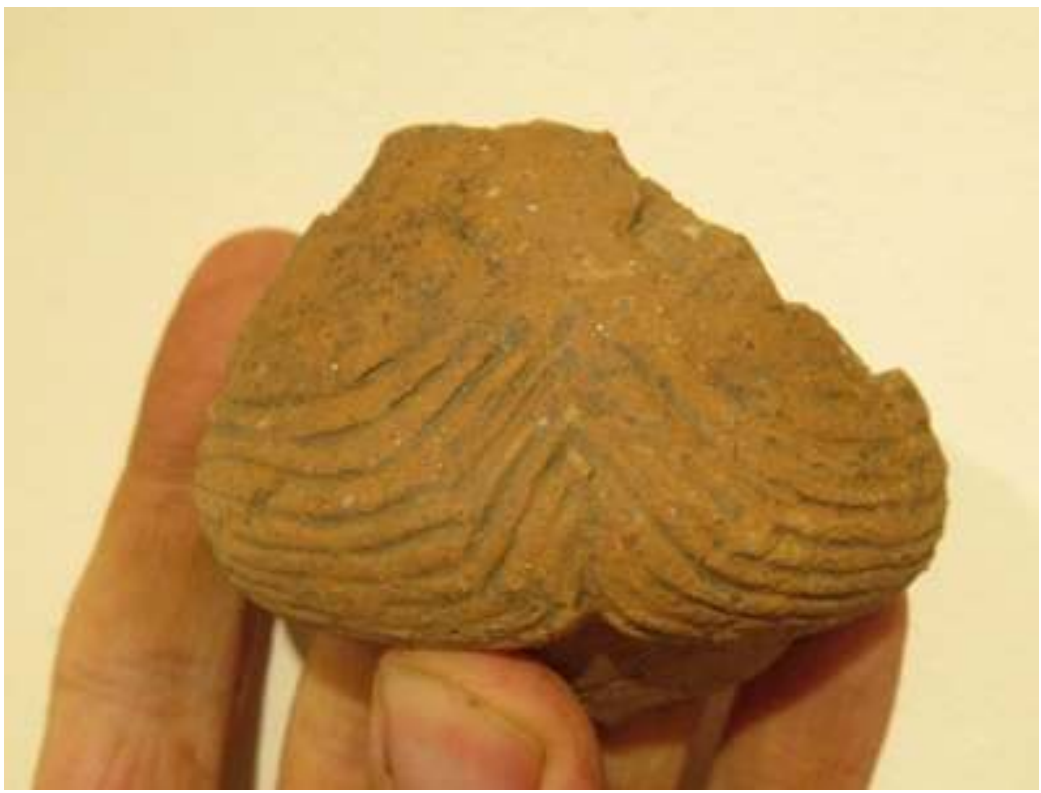

Crédit : Péter Véninger 
Fig. 22 - Tête de statuette fragmentaire témoignant de la technique de la " construction » de la chevelure à partir de pièces moulées séparément. Musée National de Reggio Calabria.

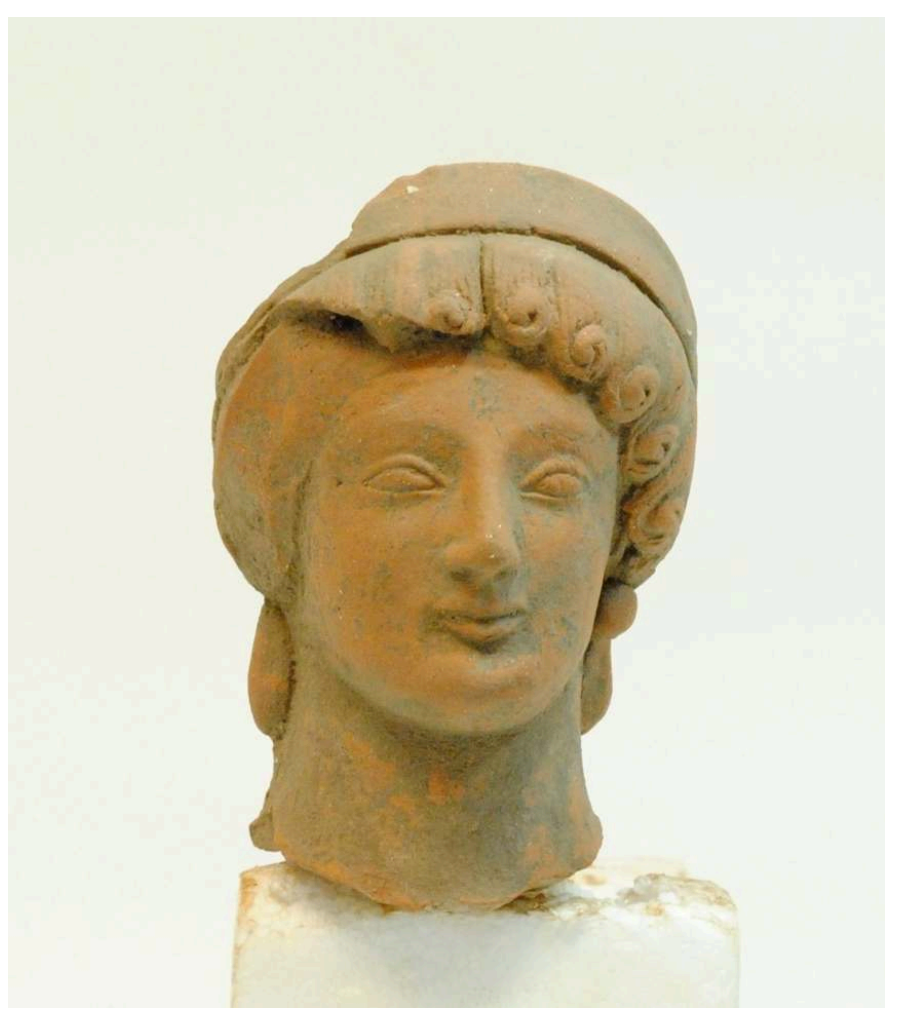

Crédit : Judit Vörös.

Enfin, nous avons observé les traces de retouche ayant pour but la correction de fautes de moulage. La poitrine d'une statuette (fig. 23) montre à la fois une tentative pour effacer une cassure horizontale, qui court à travers les plis du chiton, et des ajouts, plutôt maladroits, afin de faire ressortir les seins, sans doute jugés trop aplatis à cause du pressage insuffisant de la croûte dans le moule. Les traces, plutôt fréquentes, de corrections similaires confirment l'impression que nous avons déjà relevée auparavant, à savoir que nous avons à faire ici à une attitude très soucieuse de l'apparence finale des produits, tout en permettant des imperfections techniques au cours de la fabrication. 
Fig. 23 - Détail de statuette moulée avec traces de corrections après le moulage. Musée Archéologique de Rosarno.

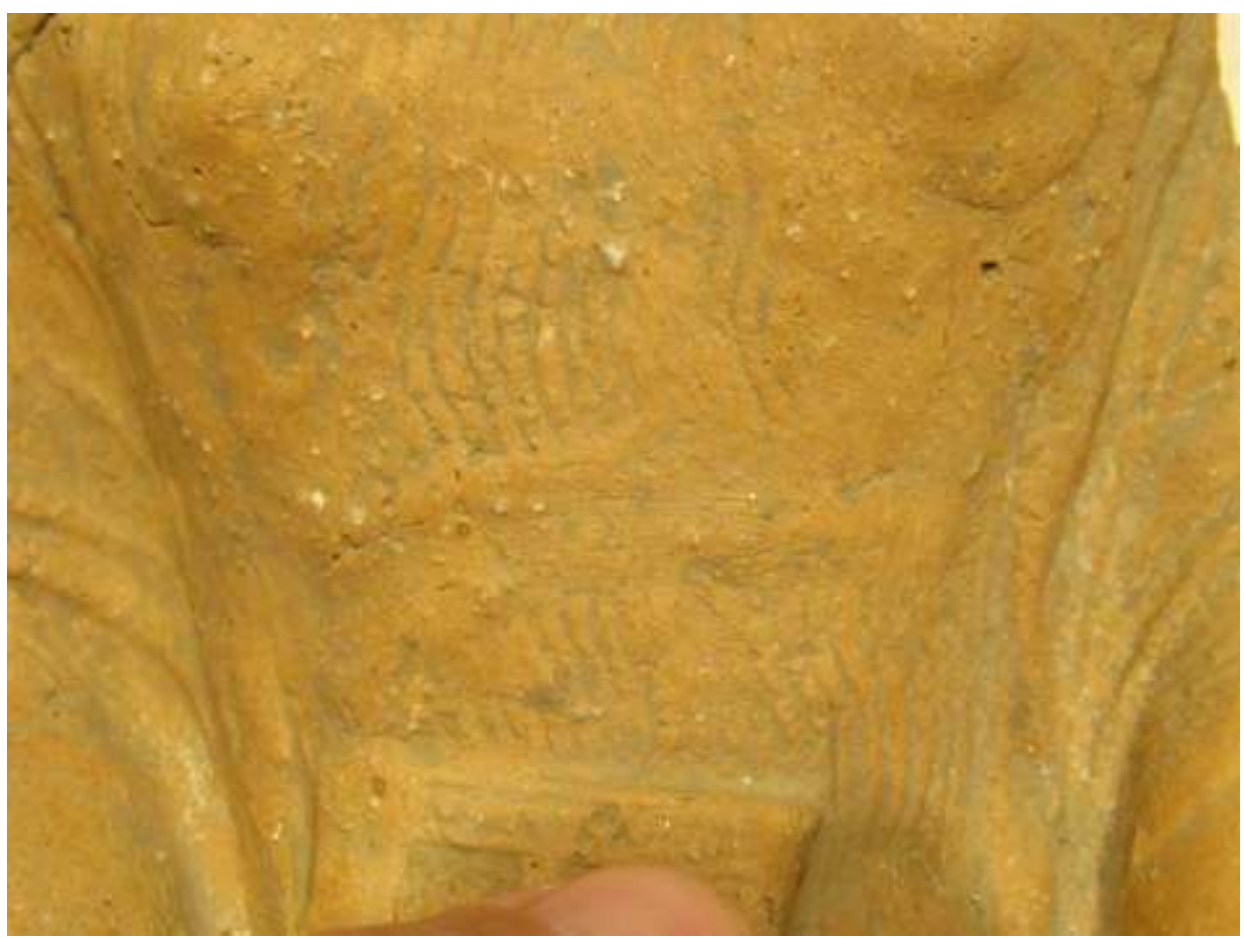

Crédit : Péter Véninger

Séchage: Nous n'avons pas détecté de trace particulière à mettre en relation avec la phase du séchage.

\section{Cuisson}

Détecter les possibles modalités de cuisson peut fournir une clé pour l'interprétation des différences les plus visibles à l'intérieur du dépôt de Calderazzo. Nous nous limitons ici à enregistrer quelques observations et en donner des explications préliminaires qui seront à vérifier et à approfondir par la suite.

Les terres cuites du dépôt montrent toutes les traits physiques de la céramique cuite à une température inférieure à celle utilisée actuellement (supérieure ou égale à $960^{\circ}$ ). Ceci est suggéré surtout par la consistance toujours relativement tendre et parfois même friable, sans pouvoir exclure, toutefois, que cela puisse dépendre aussi de l'érosion du matériel due aux conditions climatiques dans lesquelles il s'est conservé.

La couleur toujours homogène des surfaces (manque de taches de couleurs différentes et de nuances sur l'extérieur) montre que la cuisson était uniforme, et devait être exécutée au four.

40 Sans compter les diverses nuances et les pièces qui sont difficiles à classer pour le moment, deux groupes majeurs peuvent être distingués :

- Couleur «rouge brique " (Munsell 5YR 7/6 à 7/8 et 6/6 à 6/8), homogène sur toute l'épaisseur de la croûte, de consistance variable.

- Croûte stratifiée, avec deux couches claires, de la couleur pêche à l'orange (Munsell 5YR 8/2 à $8 / 4$, Munsell 7.5YR 7/4 à 7/6), avec une couche grise (de nuances variables) au milieu, de consistance plus dure. 
41 La structure stratifiée mentionnée en deuxième lieu est le résultat d'une cuisson prolongée, articulée en deux phases : une longue phase réductrice (gris) et une dernière phase oxydante, vers la fin de la cuisson, correspondant au rafraîchissement du four.

42 La coexistence de ces deux qualités de terres cuites en grandes quantités, correspondant à deux modalités différentes de cuisson, constitue l'indice le plus fort de ce que les offrandes de Calderazzo provenaient au moins de deux ateliers, probablement situés en deux endroits caractérisés par des conditions distinctes. Pour nous rapprocher de l'interprétation la plus exacte possible, il faudra mieux comprendre les circonstances susceptibles d'avoir imposé la technique qui produisait la pâte à structure stratifiée.

Un dernier groupe, quantitativement plus réduit, est composé de terres cuites à pâte jaune, extrêmement tendre et fragile. À propos de celles-ci on doit supposer que la couleur jaune correspond au résultat d'une technique de cuisson particulière plutôt qu'à la composition de l'argile (« argile jaune »)11.

\section{Conclusions}

En guise de conclusion des observations énumérées ci-dessus et des hypothèses préliminaires qu'elles nous suggèrent, il convient de souligner les points qui nous semblent être les plus prometteurs pour les enquêtes à suivre, concernant la question de l'atelier (ou des ateliers) responsable(s) de la production de ces terres cuites. Bien que nous n'ayons pas consacré un exposé particulier aux diverses formes de détérioration qui ont conditionné l'état actuel des objets, il est indispensable d'en tenir compte. La perte d'informations, due à l'état fragmentaire des pièces, aux incrustations calcaires, de sels minéraux ou autres, ainsi qu'aux pratiques de stockage et de nettoyage inadéquates, est certainement très importante. Toutefois, le grand nombre de documents disponibles nous permet de formuler des observations au moins approximatives, en nous appuyant sur la quantité plutôt significative des traces conservées.

Nous avons constaté la présence simultanée de diverses qualités de pâte dans le répertoire du dépôt de Calderazzo, pour le moins perceptibles à l'œil. Ces différences sont dues à deux facteurs majeurs, selon toute vraisemblance : la préparation de la pâte et la technique de cuisson. Quant à la première, les terres cuites du dépôt ont été faites toutes d'argiles fortement dégraissées, ce qui est à considérer comme le résultat d'un choix délibéré ou de pratiques intentionnelles. En effet, l'argile dégraissée est plus facile à traiter et moins fragile au cours des procédés du moulage, du séchage et de la cuisson - tandis qu'elle est moins appropriée au modelage, mais c'était important surtout pour la fabrication des prototypes, dont nous ne connaissons aucun exemplaire. C'était la pâte fortement dégraissée qui permettait et requérait d'utiliser l'argile à un état particulièrement tendre, c'est-à-dire très humide. D'autre part, il n'est pas vraisemblable qu'un seul atelier ait utilisé des argiles de composition très différente, à peu près durant les mêmes décennies et pour en fabriquer des objets de la même fonction, voire appartenant au même répertoire iconographique et stylistique. Surtout, il n'est pas évident que les artisans du même lieu de production soient allés chercher des argiles suffisamment dégraissées, mais de composition différente. Même si cela n'est pas à exclure complètement, il nous semble beaucoup plus probable que les 
statuettes moulées dans des argiles de compositions diverses aient été fabriquées en des lieux divers, même si elles répondaient à la même demande et reproduisaient plus ou moins le même répertoire typologique.

Dans les grandes lignes, on peut faire les mêmes observations à propos des différences de la cuisson. De ce point de vue, nous avons reconnu deux groupes majeurs (les pièces de teint homogène, oscillant parmi les nuances du rouge brique; les terres cuites de structure stratifiée, avec une couche grise bien distinguée entre deux couches rougeâtres claires) et un groupe moins significatif du point de vue quantitatif (de couleur jaune pâle, très tendre et friable). Ici, comme dans le cas de la préparation de l'argile, l'hypothèse de deux ou trois centres de production est très probable, étant donné que les différences dans les démarches suivies au cours de la cuisson devaient être liées surtout à la diversité des solutions techniques imposées par les circonstances de chaque lieu, par les conditions climatiques, géologiques et autres.

Il nous semble tout à fait probable qu'une enquête systématique, consistant à comparer les types coroplastiques, identifiés à travers l'analyse des formes, aux diverses qualités matérielles, définies sur la base des observations techniques et des futures analyses pétrographiques, pourra mieux éclairer la relation entre les diverses traditions stylistiques et les pratiques artisanales des ateliers producteurs.

En même temps il faut rappeler aussi les caractéristiques fondamentalement homogènes pour tout le matériel en ce qui concerne les techniques des artisans aux diverses étapes du moulage et de l'assemblage des statuettes, décelées surtout par la variété des fautes rencontrées et par l'absence d'autres. Pour en donner un jugement synthétique, formulé en une phrase, on pourrait dire que les producteurs des offrandes de terre cuite du dépôt étaient extrêmement soucieux de l'aspect esthétique de leurs produits, tandis qu'ils tendaient à traiter avec négligence la plupart des aspects techniques qui n'avaient pas de connexion directe avec l'apparence des surfaces externes. Les prototypes et les moules (presque tous de "première génération ») étaient aussi de très haute qualité du point de vue technique. Les statuettes étaient pour la plupart finies par l'assemblage de plusieurs parties moulées et modelées, et presque toujours retouchées ultérieurement. Il n'y a quasiment aucune trace de fautes de moulage importantes sur les surfaces, et, dans les cas où elles se présentaient par hasard, nous voyons aussi les traces de corrections immédiates. S'opposant à cette perfection formelle, nous avons pu observer toute une série d'incohérences, de négligences et de fautes sérieuses dans la technique de moulage.

Bien que ces analyses techniques soient à considérer comme préliminaires, une constatation semble s'imposer sur la base de nos observations: les démarches artisanales et l'attitude « mentale » qu'elles révèlent, apparaissent constantes à travers tout le matériel, indépendamment des diversités enregistrées dans la qualité physique des pâtes. Une certaine évolution technologique pourra être détectée en termes chronologiques, mais en ce qui concerne le corps du matériel le plus consistant, coïncidant à peu près avec les décennies $490-460$ av. J.-C., il semble que les producteurs de nos terres cuites aient partagé entre eux les mêmes prémisses culturelles ou, au moins, la même formation au métier de coroplathe. 


\section{BIBLIOGRAPHY}

Bencze 2019a = Á. Bencze, Recherches sur les terres cuites votives de Medma, dans Chronique des activités archéologiques de l'École française de Rome [En ligne], Italie du Sud, mis en ligne le 29 novembre 2019, consulté le 15 novembre 2020. URL : http://journals.openedition.org/cefr/ 3947.

Bencze 2019b = Á. Bencze, Statuette per il divino. Le officine di terrecotte votive a Medma, dans C. Malacrino, M. Cannatà (dir.), Medma. Una colonia locrese sul Tirreno, Reggio de Calabre, 2019, p. $109-125$

Bencze 2020 = Á. Bencze, Type, modelage et style. L'exemple des terres cuites du sanctuaire de Calderazzo, Medma, dans H. Aurigny, L. Rohaut (dir.), « Quand on a la terre sous l'ongle »: Le modelage dans le monde grec antique. Colloque international, 3-5 avril 2019, Aix-en-Provence, 2020, à paraître.

Miller 1983 = R. Miller, The Terracotta Votives from Medma: Cult and Coroplastic Craft in Magna Graecia, Diss. Univ. Michigan, Ann Arbor, 1983.

Miller Ammerman 1985 = R. Miller Ammerman, Medma and the exchange of Votive Terracottas, dans Papers in Italian Archaeology, 4, 1985, p. 5-19.

Miller Ammerman $1990=$ R. Miller Ammerman, Terrecotte votive: evidenze di culto e contatto culturale in Magna Grecia, dans ScAnt, 2-4, 1989-90, p. 353-362.

Muller 1997 = A. Muller, Description et analyse des productions moulées. Proposition de lexique multilingue, suggestions de méthode, dans A. Muller (dir.), Le moulage en terre cuite dans l'Antiquité. Création et production dérivée, fabrication et diffusion, Lille, p. 437-463.

Muller $2014=$ A. Muller, L'atelier du coroplathe $:$ un cas particulier dans la production céramique grecque, dans Perspective, 1, 2014, p. 68-82.

Orsi $1913^{\circ}=$ P. Orsi, Scavi di Calabria nel 1913, dans NSA, 1913, p. 4-54.

Orsi $1913 \mathrm{~b}=\mathrm{P}$. Orsi, Rosarno (Medma?). Esplorazione di un grande deposito di terrecotte ieratiche, dans NSA, 1913, p. 55-144.

Véninger 2019 = P. Véninger, Mi mindenre lehet következtetni a régészeti kerámiák szineiböl ? / What can be concluded from the coulours of the archaeological ceramics?, dans M $\Omega$ MO $\Sigma$ X. Öskoros Kutatók X. Összejövetelének konferenciakötete. Őskori technikák, őskori technológiák, Százhalombatta 2017. április 6-8., Százhalombatta, 2019, p. 410-427.

\section{NOTES}

1. Pour une introduction générale et sur les résultats des trois premières campagnes voir Bencze 2019a. Pour le moment nos recherches ne concernent que le matériel fouillé par P. Orsi au début du XX siècle : Orsi 1913a et Orsi 1913b.

2. Pour les recherches précédentes voir surtout Miller 1983, Miller Ammerman 1985 et Miller Ammerman 1990. Sur les observations et leur interprétation proposées dans le cadre de ces recherches Bencze 2019a et Bencze 2019b.

3. Les recherches de R. Miller Ammerman ont bien montré le très grand intérêt inhérent à l'observation des diverses qualités de la pâte (couleur, granulosité, dégraissants, consistance) qui compose les statuettes. En même temps, il semble aujourd'hui évident que la description de ces 
caractéristiques en elles-mêmes ne permet ni de localiser la provenance des argiles ni de les lier à des ateliers distincts. Les analyses chimiques restent, elles aussi, insuffisantes à cet égard, puisque la composition de l'argile n'était qu'un des traits caractéristiques d'un atelier, et pas le plus constant.

4. Pour la terminologie spécifique de la coroplathie nous suivons les propositions de Muller 1997.

5. Le modèle d'organisation que nous pouvons nous figurer pour ces coroplathes correspondrait à celui dont A. Muller tend à exclure pratiquement l'existence, cf. Muller 2014, p. 5-7.

6. Miller Ammerman 1983 ; Miller Ammerman 1985, p. 8 ; Bencze 2019a, section 12.

7. Cf. aussi Muller 1997, p. 440.

8. Sans doute c'est le même phénomène auquel s'est référée Miller 1985, p. 8 avec le terme de « limestone reaction rings", erroné, puisqu'il ne s'agit pas de calcaire, tandis que la brisure en «cratère » ou, autrement dit, en "anneaux concentriques" peut évoquer aussi les traces typiques d'une inclusion calcique, laquelle, tout en étant très bien connue dans la céramique antique en général, est apparemment absente à Medma/Calderazzo.

9. Bencze 2020.

10. Bencze 2020, fig. 1-3.

11. Pour la question des couleurs de la céramique antique en rapport avec les techniques de cuisson voir Véninger 2019.

\section{INDEX}

Mots-clés: Terres cuites votives, technique de fabrication, archéométrie

Geographical index: Medma

institutions Université catholique Péter Pázmány, Budapest ; École française de Rome ;

Soprintendenza Archeologia, Belle Arti e Paesaggio per la città metropolitana di Reggio Calabria $\mathrm{e}$ la provincia di Vibo Valentia (ABAP - RC-VV) ; Laboratorio SuMMA - Università Mediterranea di Reggio Calabria

\section{AUTHORS}

\section{ÁGNES BENCZE}

Catholique Péter Pázmány de Budapest (Pázmány Péter Katolikus Egyetem)

\section{PÉTER VÉNINGER}

Université des Beaux-Arts de Budapest (Képzőművészeti Egyetem) 